TRANSACTIONS OF THE

AMERICAN MATHEMATICAL SOCIETY

Volume 365, Number 8, August 2013, Pages 4041-4062

S 0002-9947(2013)05655-3

Article electronically published on April 2, 2013

\title{
ON THE IRREDUCIBILITY \\ OF THE DIRICHLET POLYNOMIAL OF AN ALTERNATING GROUP
}

\author{
MASSIMILIANO PATASSINI
}

Abstract. Given a finite group $G$ the Dirichlet polynomial of $G$ is

$$
P_{G}(s)=\sum_{H \leq G} \frac{\mu_{G}(H)}{|G: H|^{s}},
$$

where $\mu_{G}$ is the Möbius function of the subgroup lattice of $G$. This object is a member of the factorial domain of finite Dirichlet series. In this paper we prove that if $G$ is an alternating group of degree $k$ and $k \leq 4.2 \cdot 10^{16}$ or $k \geq\left(e^{e^{15}}+2\right)^{3}$, then $P_{G}(s)$ is irreducible. Moreover, assuming the Riemman Hypothesis, we prove that $P_{G}(s)$ is irreducible in the remaining cases.

An important object in number theory is the Riemann Zeta function

$$
\zeta(s)=\sum_{n \geq 1} \frac{1}{n^{s}}
$$

In the literature there are many generalizations of this object, also connected with the Euler expansion associated to $\zeta(s)$, i.e.

$$
\frac{1}{\zeta(s)}=\prod_{p \text { prime }}\left(1-\frac{1}{p^{s}}\right)
$$

A generalization was introduced by Mann ([Man96]). Given a finitely generated group $G$, Mann defined the Dirichlet series of $G$ in the following way:

$$
P_{G}(s)=\sum_{k \geq 1} \frac{a_{k}(G)}{k^{s}}, \text { where } a_{k}(G)=\sum_{H \leq G,|G: H|=k} \mu_{G}(H),
$$

where $\mu_{G}$ is the Möbius function of the subgroup lattice of $G$, which is defined inductively by $\mu_{G}(G)=1, \mu_{G}(H)=-\sum_{K>H} \mu_{G}(K)$. In particular we have that

$$
P_{\mathbb{Z}}(s)=\frac{1}{\zeta(s)}=\prod_{p \text { prime }} P_{\mathbb{Z} / p \mathbb{Z}}(s) .
$$

So, the Dirichlet series $1 / P_{G}(s)$ was called the probabilistic Zeta function of $G$. The term probabilistic is due to the probabilistic interpretation of the values of the function at the natural numbers (see Man96] for further references).

Received by the editors May 12, 2011 and, in revised form, June 11, 2011, and June 19, 2011. 2010 Mathematics Subject Classification. Primary 11M41; Secondary 11N05, 20D06, 20 E28. 
In this paper, we deal with the finite case, so we assume that $G$ is a finite group. Hence $P_{G}(s)$ is an element of the ring of finite Dirichlet series (also called Dirichlet polynomials) with integer coefficients, i.e.

$$
\mathcal{R}=\left\{\sum_{m \geq 1} \frac{a_{m}}{m^{s}}: a_{m} \in \mathbb{Z},\left|\left\{m: a_{m} \neq 0\right\}\right|<\infty\right\},
$$

which is a factorial domain (see [DLM04]). Hence it is interesting to understand the factorization of $P_{G}(s)$ in $\mathcal{R}$. Indeed, we wish to find some information about the structure of the group (e.g. the chief factors) from the knowledge of the factorization of $P_{G}(s)$.

The irreducible factors of $P_{G}(s)$ are usually called the generalized Euler factors, and their analysis could be the beginning for a number theoretical interpretation of these Dirichlet polynomials.

There are some results on the factorization of $P_{G}(s)$. Let $1=G_{0} \unlhd \cdots \unlhd G_{k}=G$ be a chief series of $G$. The Dirichlet polynomial of $G$ factors in the following way:

$$
P_{G}(s)=\prod_{i=0}^{k-1} P_{G / G_{i}, G_{i+1} / G_{i}}(s)
$$

where, for a normal subgroup $N$ of $G$, we have

$$
P_{G, N}(s)=\sum_{k \geq 1} \frac{a_{k}(G, N)}{k^{s}}, \text { where } a_{k}(G, N)=\sum_{\substack{H \leq G,|G: H|=k, N H=G,}} \mu_{G}(H) .
$$

By [DL03], the factors of ( $\dagger$ ) are independent on the choice of the chief series. Nevertheless, in general, these factors are not irreducible. It is easy to see that if $P_{G}(s)$ is irreducible, then $G / \operatorname{Frat}(G)$ is simple (see for example DLM04, Corollary 7]; Frat $(G)$ denotes the Frattini subgroup of $G$ ). But the converse is not true: for instance, $P_{\mathrm{PSL}_{2}(7)}(s)$ is reducible.

The question of the irreducibility of the Dirichlet polynomial of a simple group was proposed by N. Boston (see [Bos96]), and the first significant results were obtained in DLM04, where it was proved that if $p$ is a prime number greater than 3 , then $P_{\mathrm{Alt}_{p}}(s)$ is irreducible, and $P_{\mathrm{PSL}_{2}(p)}(s)$ is reducible if and only if $p=2^{e}-1$ with $e \equiv 3(\bmod 4)$. In a recent paper $($ Pat09]), we proved that given a simple group $G$ of Lie type, $P_{G}(s)$ is reducible if and only if $G=\mathrm{PSL}_{2}(p)$ with $p=2^{e}-1$ a prime number and $e \equiv 3(\bmod 4)$.

In this paper we study the case when $G$ is an alternating simple group, i.e. $G=\mathrm{Alt}_{k}$ and $k \geq 5$, and we prove the following.

Theorem 1. Assume that $k \geq 5$.

If $k \leq 4.2 \cdot 10^{16}$ or $k \geq\left(e^{e^{15}}+2\right)^{3}$, then $P_{\mathrm{Alt}_{k}}(s)$ is irreducible in the ring of Dirichlet finite series.

Assuming the Riemann Hypothesis, $P_{\mathrm{Alt}_{k}}(s)$ is irreducible in the ring of Dirichlet finite series.

As explained in Section 1, the ring $\mathcal{R}$ is isomorphic to a ring of multivariate polynomials with integer coefficients. Essentially, we study a problem of reducibility of a multivariate polynomial. Using some knowledge of group theory, we reduce the problem to a number theoretical conjecture, which is true conditionally. 
The factors of the factorization $(\dagger)$ can be described in terms of Dirichlet polynomials of monolithic primitive groups. We recall that a group $G$ is monolithic primitive if there exists a maximal subgroup $M$ such that $\bigcap_{g \in G} M^{g}=1$ and $G$ has a unique minimal normal subgroup. Let $G$ be a finite group, and let $N$ be a minimal normal subgroup of $G$. It is possible to define a monolithic primitive group associated with $N$ in the following way (see [DL03]):

$$
L_{N}=\left\{\begin{array}{cc}
G / C_{G}(N) & \text { if } N \text { is not abelian, } \\
G / C_{G}(N) \ltimes N & \text { otherwise. }
\end{array}\right.
$$

Clearly $N$ is (isomorphic to) the unique minimal normal subgroup of $L$, so $N=$ $\operatorname{soc}\left(L_{N}\right)$ (i.e. $N$ is the socle of $L_{N}$ ). Under these assumptions, there exists an integer $\alpha$ such that

$$
P_{G, N}(s)=P_{L_{N}, \operatorname{soc}\left(L_{N}\right)}(s)+\frac{\alpha}{|N|^{s}}
$$

The above remark shows that the study of the irreducibility of the factors in $(\dagger)$ is strictly connected with the study of the irreducibility of $P_{G, \operatorname{soc}(G)}(s)$, where $G$ is a monolithic primitive group.

Thanks to a result of [Ser08, we obtain the following.

Theorem 2. Assume that $k \geq 5$. Let $G$ be a primitive monolithic group with a simple component isomorphic to $\mathrm{Alt}_{k}$. Moreover assume that if $k=6$, then $N_{G}(S) / C_{G}(S)$ is contained in the Symmetric group $\operatorname{Sym}_{k}$.

If $k \leq 4.2 \cdot 10^{16}$ or $k \geq\left(e^{e^{15}}+2\right)^{3}$, then $P_{G, \operatorname{soc}(G)}(s)$ is irreducible in the ring of Dirichlet finite series.

If the Riemann Hypothesis holds, then $P_{G, \operatorname{soc}(G)}(s)$ is irreducible in the ring of Dirichlet finite series.

Using our techniques, when $k=6$ and $N_{G}(S) / C_{G}(S) \neq \mathrm{Alt}_{6}$ (that is, $N_{G}(S) / C_{G}(S) \cong \mathrm{PGL}_{2}(9), M_{10}$ or $\left.\operatorname{Aut}\left(\mathrm{Alt}_{6}\right)\right)$, we have not enough information to decide whether $P_{G, \operatorname{soc}(G)}(s)$ is irreducible or not. However, direct computations show that $P_{G, \operatorname{soc}(G)}(s)$ is irreducible if $G \cong \mathrm{PGL}_{2}(9), M_{10}$ or $\operatorname{Aut}\left(\operatorname{Alt}_{6}\right)$.

The result we obtain has an interesting consequence, as the following theorem shows.

Theorem 3. Let $G$ and $H$ be two finite groups such that $P_{G}(s)=P_{H}(s)$. Assume that the composition factors of $G$ and $H$ are alternating groups which satisfy the hypothesis of Theorem 2 . Then $G$ and $H$ have the same non-Frattini chief factors.

In Section 1, we outline the strategy of the proof and we introduce notation and lemmas that we shall use throughout the paper.

In Section 2] we state and attack a number theoretical conjecture, which we believe to be true. This conjecture is crucial in order to prove our main result.

Section 3 is devoted to the proof of Theorem 2 .

In Section 4 we prove that it is possible to recognize the number of factors of the socle of a primitive monolithic group $L$ from the knowledge of the Dirichlet polynomial $P_{L, \operatorname{soc}(L)}(s)$.

Finally, in Section [5] we prove Theorem 3 


\section{Strategy of the proof, some definitions and lemmas}

In the sequel, we outline the strategy of the proof in the simple case, i.e. $G=$ $\mathrm{Alt}_{k}, k \geq 5$. Let $\pi$ be a set of primes, and let $\pi^{\prime}$ be the set of prime numbers that does not lie in $\pi$.

The first ingredient is the ring homomorphism given by

$$
\begin{array}{lll}
\eta_{\pi}: \quad \mathcal{R} & \rightarrow & \mathcal{R}_{\pi^{\prime}} \\
& f(s)=\sum_{n \in \mathbb{N}} \frac{a_{n}}{n^{s}} & \mapsto
\end{array}
$$

where

$$
b_{n}=\left\{\begin{array}{cc}
a_{n} & \text { if } n \text { is a } \pi^{\prime} \text { number } \\
0 & \text { otherwise, }
\end{array}\right.
$$

$\pi$ is a set of prime numbers, and $\mathcal{R}_{\pi^{\prime}}$ is the image of $\mathcal{R}$ through $\eta_{\pi}$.

By [DLM04, Lemma 2], in order to prove that a Dirichlet polynomial $f(s)$ is irreducible, it is enough to find a prime number $r$ such that

(1) $f^{(r)}(s)$ is irreducible;

(2) if $r \mid n$ and $a_{n}(f(s)) \neq 0$, then $|n|_{r}=|f(s)|_{r}$ (where $|f(s)|_{r}=\max \left\{|m|_{r}\right.$ : $\left.a_{m} \neq 0\right\}$, for $\left.f(s)=\sum_{n \in \mathbb{N}} \frac{a_{n}}{n^{s}}\right)$;

(3) $\left(f(s), f^{(r)}(s)\right)=1$.

It turns out that the most difficult condition to check is the third. However, if $f^{(r)}(s)$ is irreducible, it is easy to see that the following condition,

(3') the inequality

$$
\frac{M\left(f^{(r)}(s)\right)}{m\left(f^{(r)}(s)\right)}>\frac{M\left(f(s)-f^{(r)}(s)\right)}{m\left(f(s)-f^{(r)}(s)\right)}
$$

holds, where $m(g(s))=\min \left\{k: a_{k} \neq 0\right\}$ and $M(g(s))=\max \left\{k: a_{k} \neq 0\right\}$ for a Dirichlet polynomial $g(s)=\sum_{k \geq 1} \frac{a_{k}}{k^{s}}$,

implies (3).

The second ingredient is a remark. Let $r$ be a prime number such that $\frac{k}{2}<r<$ $k-2$. Then the index of a non-zero coefficient of $P_{G}^{(r)}(s)$ is of the form

$$
\frac{k !}{i_{1} ! \cdots i_{j} !\left(k-i_{1}-\cdots-i_{j}\right) !},
$$

for some $j \in \mathbb{N}$ and $i_{1}, \ldots, i_{j} \in \mathbb{N}$ such that $i_{1}+\cdots+i_{j} \leq \frac{k-1}{2}$ (see Lemma 19 and Proposition 20 for a precise justification). Let $l$ be a positive integer. We say that a Dirichlet polynomial is of type $T_{l}$ if the index of the non-zero coefficient are of the form $(*)$ and the maximum value of a sum $i_{1}+\cdots+i_{j}$ is $l$. It turns out that $P_{G}^{(r)}(s)$ is of type $T_{k-r}$.

Now, assume that there exists two prime numbers $p_{1}, p_{2}$ such that $k / 2<p_{2}<$ $p_{1}<k-2$. We construct two finite sequences $\left\{r_{i}\right\}_{i \leq \iota}$ (of prime numbers) and $\left\{a_{i}\right\}_{i \leq \iota}$ (of natural number) such that $r_{i} \mid k-a_{i}+1, r_{\iota}=p_{2}$ and $a_{1} \in\{3,4\}$. This determines a sequence $\left\{T_{a_{i}-1}(s)\right\}_{i \leq \iota}$ of Dirichlet polynomials of type (respectively) $T_{a_{1}-1}, \ldots, T_{a_{\iota}-1}$ such that $T_{a_{i}-1}(s)=P_{G}^{\left(r_{i}, \ldots, r_{\iota}\right)}(s)$ for $1 \leq i \leq \iota-1$ (so the sequence $\left\{a_{i}\right\}_{i \leq \iota}$ is increasing). In particular, note that $T_{a_{i+1}-1}^{\left(r_{i}\right)}(s)=T_{a_{i}-1}(s)$ for $1 \leq i \leq$ $\iota-1$.

Our aim is to prove that $T_{a_{\iota}-1}(s)=P_{G}^{\left(r_{\iota}\right)}(s)=P_{G}^{\left(p_{2}\right)}(s)$ is irreducible. Arguing by induction, we want to show that $T_{a_{i}-1}(s)$ is irreducible for $1 \leq i \leq \iota$. We try to verify that the conditions (1), (2), and (3)' hold. With some exceptions, 
the polynomial $T_{a_{1}-1}(s)$ is irreducible. By induction, since $T_{a_{i+1}-1}^{\left(r_{i}\right)}(s)=T_{a_{i}-1}(s)$ is irreducible, condition (1) is fulfilled (with $r=r_{i}$ and $f(s)=T_{a_{i+1}-1}(s)$ ). To satisfy condition (2), we ask that $r_{i} \geq a_{i}$ and $2 a_{i} \geq a_{i+1}$. Finally, condition (3)' is equivalent to

$$
\frac{M\left(T_{a_{i}-1}(s)\right)}{m\left(T_{a_{i}-1}(s)\right)}>\frac{M\left(T_{a_{i+1}-1}(s)-T_{a_{i}-1}(s)\right)}{m\left(T_{a_{i+1}-1}(s)-T_{a_{i}-1}(s)\right)} .
$$

Some information on the alternating groups show that the above inequality is equivalent to

$$
\left(\begin{array}{c}
k \\
a_{i}
\end{array}\right)>\frac{\left(k-a_{i}+1\right) !}{\left(k-a_{i+1}+1\right) !}
$$

By the above discussion, in order to construct the sequences $\left\{r_{i}\right\}_{i \leq \iota}$ and $\left\{a_{i}\right\}_{i \leq \iota}$, it is sufficient to find for each pair $(k, a)$ of natural number, with $3 \leq a \leq k-p_{2}$, a positive integer $h \leq a$ such that $\left(\begin{array}{l}k \\ a\end{array}\right)>\frac{(k-a+1) !}{(k-a-h+1) !}$, and the largest prime divisor of $\frac{(k-a) !}{(k-a-h) !}$ is greater than $a+h$. This is the number theoretical problem we try to attack in Section 2 (see Conjecture 7). For the precise argument see the proof of Theorem 23.

When we know that $P_{G}^{\left(p_{2}\right)}(s)$ is irreducible, the proof of the irreducibility of $P_{G}(s)$ can be easily completed (see the end of the proof of Theorem [23).

Now, we introduce some notation we shall use throughout the paper.

- Let

$$
\mathcal{R}^{\prime}=\left\{\sum_{m \geq 1} \frac{a_{m}}{m^{s}}: a_{m} \in m \mathbb{Z},\left|\left\{m: a_{m} \neq 0\right\}\right|<\infty\right\},
$$

which is a subring of $\mathcal{R}$. Let $\mathcal{R}_{\pi}^{\prime}=\mathcal{R}^{\prime} \cap \mathcal{R}_{\pi}$. We define the map

$$
\Psi: \mathcal{R}_{\pi}^{\prime} \rightarrow \mathbb{Z}\left[\mathbf{X}_{\pi}\right]
$$

given by $\Psi\left(p^{1-s}\right)=x_{p}$ for each $p \in \pi$, where $\mathbf{X}_{\pi}$ is the set of commuting indeterminates $\left\{x_{r}: r \in \pi\right\}$. Clearly, $\Psi$ is a ring isomorphism, hence $\mathcal{R}_{\pi}^{\prime}$ and $\mathcal{R}^{\prime}$ are factorial domains.

- Let $k$ be a positive integer. We let $\mathrm{p}(k)$ be the set of prime numbers that divides $k$. If $H$ is a finite group, we let $\mathrm{p}(H)=\mathrm{p}(|H|)$. If $p$ is a prime number, then we let $|k|_{p}$ be the $p$-part of $k$, i.e. $|k|_{p}=p^{i}$ where $p^{i}$ divides $n$ but $p^{i+1}$ does not divide $k$.

- Let $f(s)=\sum_{k>1} \frac{a_{k}}{k^{s}}$ be an element of $\mathcal{R}$, and let $r$ be a prime number. We let $a_{k}(f(s))=a_{k}$. Moreover, $|f(s)|_{r}=\max \left\{|k|_{r}: a_{k}(f(s)) \neq 0\right\}$. Finally $\mathrm{p}(f(s))=\mathrm{p}\left(\operatorname{lcm}\left\{k: a_{k}(f(s)) \neq 0\right\}\right)$.

We will use repeatedly, often without mention, the following results on the Möbius function of the subgroup lattice of a finite group $G$.

Lemma 4 (See [Hal36]). Let $G$ be a finite group and $H$ a subgroup of $G$. If $\mu_{G}(H) \neq 0$, then $H$ is intersection of maximal subgroups of $G$.

Lemma 5 (HIÖ89, Theorem 4.5). Let $G$ be a finite group and $H$ a subgroup of G. The index $\left|N_{G}(H): H\right|$ divides $\mu_{G}(H)\left|G: H G^{\prime}\right|$.

Note that if $X$ is an almost simple group with socle $S$, then clearly $S \leq G^{\prime}$, and so, for a subgroup $H \leq G$ such that $H S=G$, we have that $|G: H|$ divides $\mu_{G}(H)\left|G: N_{G}(H)\right|$. This implies that $k$ divides $a_{k}(G, S)$ for each $k \in \mathbb{N}-\{0\}$. 
Finally, another useful result is the following, which shows a connection between the Dirichlet polynomial of a primitive monolithic group and the Dirichlet polynomial of an almost simple group.

Theorem 6 (See [Ser08], Theorem 5). Let $G$ be a primitive monolithic group with a simple component $S$. Let $X=N_{G}(S) / C_{G}(S)$ and assume that $S \leq X$. Let $n=\left|G: N_{G}(S)\right|$. We have that

$$
P_{G, \operatorname{soc}(G)}^{(r)}(s)=P_{X, S}^{(r)}(n s-n+1)
$$

for each prime divisor $r$ of the order of $S$.

\section{A number theoretical Result}

This section is devoted to the attack on Conjecture 7 Indeed we will prove it in many cases.

For the rest of the paper, let $k$ be a positive integer, let $p_{1}$ be the largest prime number less than or equal to $k-3$, and let $p_{2}$ be the largest prime number less than $p_{1}$. Moreover assume that $\frac{k}{2}<p_{2}<p_{1}<k-2$. This implies that $k \geq 16$. (Using $\left[\mathrm{Nag} 52\right.$, there exists a prime number in the interval $\left[x, \frac{6}{5} x\right]$ for $x \geq 25$; so there are at least two prime numbers in the interval $\left[\frac{k}{2}+1, k-3\right]$ for $k \geq 48$ and we can check directly the result for $k<48$.)

We say that a pair $(k, a)$ is admissible if $k \geq 16,(k, a) \notin\{(19,3),(20,4),(21,5)$, $(55,5),(84,3)\}$ and $3 \leq a \leq k-p_{2}$. In particular, since $p_{2}>k / 2$, we have that $a<k / 2$.

Now we can state the conjecture we want to attack.

Conjecture 7. Let $(k, a)$ be an admissible pair. Then there exists a positive integer $h \leq a$ such that

(C1) $\left(\begin{array}{l}k \\ a\end{array}\right)>\frac{(k-a+1) !}{(k-a-h+1) !}$,

(C2) $\max \mathrm{p}\left(\frac{(k-a) !}{(k-a-h) !}\right)>a+h$, i.e. the largest prime divisor of $\frac{(k-a) !}{(k-a-h) !}$ is greater than $a+h$.

Let us introduce some definitions. If $(k, a)$ is an admissible pair, we say that $(k, a, h)$ satisfies (C1) (resp. (C2)) if the numbers $k, a, h$ satisfy (C1) (resp. (C2)). If $(k, a)$ is an admissible pair, we say that $(k, a)$ satisfies (C1) (resp. (C2)) if there exists $1 \leq h \leq a$ such that $(k, a, h)$ satisfies (C1) (resp. (C2)). We say that $k$ satisfies (C1) (resp. (C2)) if for each $a \in\left\{3, \ldots, k-p_{2}\right\}$ such that $(k, a)$ is admissible, the pair $(k, a)$ satisfies $(\mathrm{C} 1)$ (resp. (C2)). If $k$ (resp. $(k, a))$ satisfies both $(\mathrm{C} 1)$ and $(\mathrm{C} 2)$, we say that $k$ (resp. $(k, a))$ satisfies $(\mathrm{C})$.

In the next two lemmas we prove some sufficient conditions for $(k, a, h)$ to satisfy $(\mathrm{C} 1)$ and $(\mathrm{C} 2)$. Our aim is to obtain a result of this type: suppose that $\left(k_{0}, a_{0}\right)$ satisfies (C) under some conditions, then also $\left(k, a_{0}\right)$ satisfies (C) if $k \geq k_{0}$ (see Corollary 10 and Proposition 11).

Lemma 8. Let $k$ and $a$ be positive integers and let $h(k, a)=\frac{\log \left(\begin{array}{l}k \\ a\end{array}\right)}{\log (k-a+1)}$. If $h$ is a positive integer such that $h \leq h(k, a)$, then $(k, a, h)$ satisfies (C1). Moreover we have $h(k, a) \leq h(k+1, a)$ for each $k \in \mathbb{N}$ such that $1 \leq a<k$. 
Proof. Note that $k-a+1<\left(\begin{array}{l}k \\ a\end{array}\right)$ for $1<a<k$. Clearly $(k, a, 1)$ satisfies (C1). Let $2 \leq h \leq \frac{\log \left(\begin{array}{l}k \\ a\end{array}\right)}{\log (k-a+1)}$ be an integer. Then

$$
\frac{(k-a+1) !}{(k-a-h+1) !}<(k-a+1)^{h} \leq\left(\begin{array}{l}
k \\
a
\end{array}\right)
$$

hence $(k, a, h)$ satisfies $(\mathrm{C} 1)$.

Note that $h(k, a) \leq h(k+1, a)$ is equivalent to

$$
\frac{\log \left(\begin{array}{c}
k+1 \\
a
\end{array}\right)}{\log \left(\begin{array}{l}
k \\
a
\end{array}\right)} \geq \frac{\log (k-a+2)}{\log (k-a+1)} .
$$

Since $\left(\begin{array}{c}k+1 \\ a\end{array}\right)=\left(\begin{array}{l}k \\ a\end{array}\right) \frac{k+1}{k-a+1}$, the previous inequality is equivalent to

$$
\log \left(\begin{array}{l}
k \\
a
\end{array}\right) \leq \frac{\log \left(\frac{k+1}{k-a+1}\right) \log (k-a+1)}{\log \left(\frac{k-a+2}{k-a+1}\right)}
$$

Now we prove (8.1) by induction on $k$. If $k=2$, then $a=1$ and the claim is clear. Assume that $k \geq 3$. By induction, we have that

$$
\begin{gathered}
\log \left(\begin{array}{c}
k+1 \\
a
\end{array}\right)=\log \left(\left(\begin{array}{l}
k \\
a
\end{array}\right) \frac{k+1}{k-a+1}\right) \leq \frac{\log \left(\frac{k+1}{k-a+1}\right) \log (k-a+1)}{\log \left(\frac{k-a+2}{k-a+1}\right)} \\
+\log \left(\frac{k+1}{k-a+1}\right)=\frac{\log \left(\frac{k+1}{k-a+1}\right) \log (k-a+2)}{\log \left(\frac{k-a+2}{k-a+1}\right)} .
\end{gathered}
$$

In order to complete the proof, it is enough to show that the function

$$
f(k)=\frac{\log \left(\frac{k}{k-a}\right)}{\log \left(\frac{k-a+1}{k-a}\right)}
$$

is increasing if $k>a$. Note that $f^{\prime}(k) \geq 0$ if and only if

$$
\left(1+\frac{a}{k-a}\right)^{k} \geq\left(1+\frac{1}{k-a}\right)^{a(k-a+1)}
$$

i.e.

$$
\left(1+\frac{1}{\frac{k-a}{a}}\right)^{\frac{k-a}{a}+1} \geq\left(1+\frac{1}{k-a}\right)^{k-a+1},
$$

which holds since the map $y \mapsto\left(1+\frac{1}{y}\right)^{y+1}$ is decreasing for $y>0$.

Lemma 9. Let $k, a, h$ be positive integers such that $k>2 a \geq 2 h$, and let $f(k, a, h)=$ $\left(\begin{array}{c}k-a \\ h\end{array}\right)-(k-a)^{\pi(a+h)}$, where $\left.\pi:\right] 0, \infty[\rightarrow \mathbb{N}$ is the prime counting function, defined by $\pi(x)=\mid\{p: p \leq x, p$ prime number $\} \mid$.

(1) If $f(k, a, h)>0$, then $\max \mathrm{p}\left(\frac{(k-a) !}{(k-a-h) !}\right)>a+h$ (i.e. $(k, a, h)$ satisfies (C2)).

(2) If $h \geq \pi(a+h)$ and $f(k, a, h) \geq 0$, then $f(k+1, a, h) \geq f(k, a, h)$. 
Proof. Let us prove (1). Assume that $\max \mathrm{p}\left(\frac{(k-a) !}{(k-a-h) !}\right) \leq a+h$. Then also $\max \mathrm{p}\left(\left(\begin{array}{c}k-a \\ h\end{array}\right)\right) \leq a+h$. By Erd34, we have that $\left|\left(\begin{array}{c}n \\ i\end{array}\right)\right|_{p} \leq n$ for $p$ a prime number, $n$ and $i$ positive integer, $n \geq i$. Thus we obtain

$$
\left(\begin{array}{c}
k-a \\
h
\end{array}\right)=\prod_{p \text { prime }, p \leq a+h}\left|\left(\begin{array}{c}
k-a \\
h
\end{array}\right)\right|_{p} \leq(k-a)^{\pi(a+h)},
$$

hence we have (1).

Now we prove (2). The inequality $f(k+1, a, h) \geq f(k, a, h)$ is equivalent to

$$
\left(\begin{array}{l}
k-a \\
h-1
\end{array}\right) \geq(k+1-a)^{\pi(a+h)}-(k-a)^{\pi(a+h)} .
$$

Since $f(k, a, h) \geq 0$, we have that

$$
\left(\begin{array}{c}
k-a \\
h-1
\end{array}\right)=\left(\begin{array}{c}
k-a \\
h
\end{array}\right) \frac{h}{k-a-h+1} \geq(k-a)^{\pi(a+h)} \frac{h}{k-a-h+1} .
$$

In order to prove (9.1) it is enough to show that

$$
(k-a)^{\pi(a+h)} \frac{h}{k-a-h+1} \geq(k+1-a)^{\pi(a+h)}-(k-a)^{\pi(a+h)},
$$

which is equivalent to

$$
\left(1-\frac{1}{k-a+1}\right)^{\pi(a+h)} \geq 1-\frac{h}{k-a+1} .
$$

By the Bernoulli inequality, we obtain

$$
\left(1-\frac{1}{k-a+1}\right)^{\pi(a+h)} \geq 1-\frac{\pi(a+h)}{k-a+1} .
$$

Moreover, since $h \geq \pi(a+h)$, we get

$$
1-\frac{\pi(a+h)}{k-a+1} \geq 1-\frac{h}{k-a+1},
$$

so (9.2) holds.

Corollary 10. Let $(k, a)$ be an admissible pair, and let $h(k, a)=\left\lfloor\frac{\log \left(\begin{array}{l}k \\ a\end{array}\right)}{\log (k-a+1)}\right\rfloor$ and $f(k, a, h)=\left(\begin{array}{c}k-a \\ h\end{array}\right)-(k-a)^{\pi(a+h)}$. Let $k_{0}, a_{0} \in \mathbb{N}, k_{0}>a_{0} \geq 3$ be such that

(1) $h\left(k_{0}, a_{0}\right) \geq \pi\left(h\left(k_{0}, a_{0}\right)+a_{0}\right)$,

(2) $f\left(k_{0}, a_{0}, h\left(k_{0}, a_{0}\right)\right)>0$.

Then each element of the set $\left\{\left(k, a_{0}, h\left(k_{0}, a_{0}\right)\right): k \geq k_{0}, k \in \mathbb{N}\right\}$ satisfies $(\mathrm{C})$.

Proof. By Lemma 8, we have that $\left(k, a_{0}, h\left(k_{0}, a_{0}\right)\right)$ satisfies $(\mathrm{C} 1)$ for $k \geq k_{0}$.

By Lemma 9(2), since $h\left(k_{0}, a_{0}\right) \geq \pi\left(h\left(k_{0}, a_{0}\right)+a_{0}\right)$ and $f\left(k_{0}, a_{0}, h\left(k_{0}, a_{0}\right)\right)>0$, we get $f\left(k, a_{0}, h\left(k_{0}, a_{0}\right)\right)>0$ for each $k \geq k_{0}, k \in \mathbb{N}$. Thus, by Lemma 9(1), we have that $\left(k, a_{0}, h\left(k_{0}, a_{0}\right)\right)$ satisfies $(\mathrm{C} 2)$ for $k \geq k_{0}$.

Proposition 11. Let $(k, a)$ be an admissible pair, and let $\theta_{0} \in(0,1)$ be the solution of the equation $\frac{e^{\frac{2-\theta}{(1-\theta)^{2}}+1.12}}{2-\theta}=\frac{1}{\theta}$. Let $\theta_{0}<\theta<1$, and let $\frac{e^{\frac{2-\theta}{(1-\theta)^{2}}+1.12}}{2-\theta}<a \leq k^{\theta-\frac{1}{a}}$. If $\left(k^{\prime}, a\right)$ is an admissible pair such that $k^{\prime} \geq k$, then $\left(k^{\prime}, a\right)$ satisfies $(\mathrm{C})$. 
Proof. Note that the function $x \mapsto \frac{e^{\frac{2-x}{(1-x)^{2}}+1.12}}{2-x}$ is increasing for $0<x<1$. So, it turns out that $\theta_{0} \approx 0.068$. Moreover we have $a(1-\theta)>1$, since

$$
a(1-\theta)>\frac{(1-\theta) e^{\frac{2-\theta}{(1-\theta)^{2}}+1.12}}{2-\theta}=\frac{e^{1.12}}{1-\theta} \frac{e^{\frac{2-\theta}{(1-\theta)^{2}}}}{\frac{2-\theta}{1-\theta}}>\frac{e^{1.12}}{1-\theta}>1 .
$$

Moreover, using the definition of $\theta_{0}$, we get $\theta-\frac{1}{a}>0$.

We prove that $(k, a, h)$ satisfies $(\mathrm{C} 1)$ for $h=\lfloor a(1-\theta)+1\rfloor$. Since $a(1-\theta) \leq h \leq$ $a(1-\theta)+1$, there exists $\theta^{\prime} \in\left[\theta-\frac{1}{a}, \theta\right]$ such that $h=a\left(1-\theta^{\prime}\right)$. Since $a \leq k^{\theta-\frac{1}{a}} \leq k^{\theta^{\prime}}$, we have that

$$
k^{\frac{1}{1-\theta^{\prime}}} \geq a^{\frac{1}{1-\theta^{\prime}}} k \geq a^{\frac{1}{1-\theta^{\prime}}}(k-a+1)
$$

and it follows that

$$
a\left(1-\theta^{\prime}\right) \leq \frac{\log \left(\frac{k}{a}\right)^{a}}{\log (k-a+1)} \leq \frac{\log \left(\begin{array}{l}
k \\
a
\end{array}\right)}{\log (k-a+1)}
$$

since $\left(\frac{k}{a}\right)^{a} \leq\left(\begin{array}{l}k \\ a\end{array}\right)$. Thus, by Lemma 8, $\left(k^{\prime}, a, h\right)$ satisfies $(\mathrm{C} 1)$ for $h=a\left(1-\theta^{\prime}\right)$ and $k^{\prime} \geq k$.

Now, let us prove that $(k, a, h)$ satisfies $(\mathrm{C} 2)$ for $h=a\left(1-\theta^{\prime}\right)$. We have

$$
\left(\begin{array}{c}
k-a \\
a\left(1-\theta^{\prime}\right)
\end{array}\right) \geq\left(\frac{k-a}{a\left(1-\theta^{\prime}\right)}\right)^{a\left(1-\theta^{\prime}\right)} .
$$

Note that the map $x \mapsto\left(1-(1-x)^{\frac{1}{x}}\right)^{\frac{1}{x-1}}$ is increasing for $0<x<1$, so we get that $\left(1-\left(1-\theta^{\prime}\right)^{\frac{1}{\theta^{\prime}}}\right)^{\frac{1}{\theta^{\prime}-1}} \leq e \leq k$. Hence $\left(k-k^{\theta^{\prime}}\right)^{\theta^{\prime}} \geq k^{\theta^{\prime}}\left(1-\theta^{\prime}\right)$, thus $(k-a)^{\theta^{\prime}} \geq a\left(1-\theta^{\prime}\right)$. It follows that

$$
\left(\frac{k-a}{a\left(1-\theta^{\prime}\right)}\right)^{a\left(1-\theta^{\prime}\right)} \geq(k-a)^{a\left(1-\theta^{\prime}\right)^{2}} .
$$

By Lemma 9, in order to prove that $(k, a, h)$ satisfies $(\mathrm{C} 2)$, it is enough to show that $f\left(k, a, a\left(1-\theta^{\prime}\right)\right)>0$. So it remains to show that

$$
a\left(1-\theta^{\prime}\right)^{2}>\pi\left(a\left(2-\theta^{\prime}\right)\right) \text {. }
$$

Since we assumed that $a>\frac{e^{\frac{2-\theta}{(1-\theta)^{2}}+1.12}}{2-\theta}$ and the map $x \mapsto \frac{e^{\frac{2-x}{(1-x)^{2}}+1.12}}{2-x}$ is increasing for $0<x<1$, we get $a>\frac{e^{\frac{2-\theta^{\prime}}{\left(1-\theta^{\prime}\right)^{2}}+1.12}}{2-\theta^{\prime}}$, i.e.

$$
a\left(1-\theta^{\prime}\right)^{2}>\frac{a\left(2-\theta^{\prime}\right)}{\log \left(a\left(2-\theta^{\prime}\right)\right)-1.12} .
$$

By [Dus10] and some direct computation, we have that

$$
\pi(x) \leq \frac{x}{\log (x)-1.12}
$$

for $x \geq 4$. Since $a\left(2-\theta^{\prime}\right)>4$ we get

$$
\pi\left(a\left(2-\theta^{\prime}\right)\right) \leq \frac{a\left(2-\theta^{\prime}\right)}{\log \left(a\left(2-\theta^{\prime}\right)\right)-1.12} .
$$

Combining the last inequality with (11.2) we obtain (11.1).

Note that the inequality (11.1) implies that $h \geq \pi(a+h)$, hence $f\left(k^{\prime}, a, h\right) \geq$ $f(k, a, h)>0$, thus $\left(k^{\prime}, a, h\right)$ satisfies $(\mathrm{C} 2)$ for $k^{\prime} \geq k$.

When $a$ is small, we can check the conjecture directly. 
Proposition 12. Let $3 \leq a \leq 13$. If $(k, a)$ is admissible, then $(k, a)$ satisfies $(\mathrm{C})$.

Proof. Note that $4 \leq a+h \leq 26$, since $1 \leq h \leq a$. For a contradiction, assume that $(k, a)$ does not satisfy $(\mathrm{C} 2)$. Then we have that $\mathrm{p}((k-a) \cdots(k-a-h+1))$ $\subseteq \mathrm{p}((a+h) !)$. In Leh64, Table 1A], an explicit list of $n \in \mathbb{N}$ such that $\mathrm{p}(n(n-1))$ $\subseteq \mathrm{p}(m !)$ for $m \leq 42$ is given. A direct computation shows that the result holds.

The next three results concern the distribution of prime numbers in short intervals.

Lemma 13. Let $1500 \leq x \leq 4,2 \cdot 10^{16}$. Then $[x-\sqrt{x}, x]$ contains at least two prime numbers.

Proof. The maximal prime gap up to a certain number $n$ is the maximum of the difference between two consecutive prime numbers less than or equal to $n$. By NN03, the maximal prime gap for $x \leq 4.2 \cdot 10^{16}$ is less than 1184 . Thus, there exist at least two primes in the interval $[x-\sqrt{x}, x]$ if $x \geq(2 \cdot 1184)^{2}=5607424$ and $x \leq$ $4.2 \cdot 10^{16}$. By LP67 the maximal prime gap for $x \leq 10^{7}$ is less than 180 . Thus, there exist at least two primes in the interval $[x-\sqrt{x}, x]$ if $x \geq(2 \cdot 180)^{2}=129600$ and $x \leq 10^{7}$. By [LP67] the maximal prime gap for $x \leq 155000$ is less than 86 . Thus, there exist at least two primes in the interval $[x-\sqrt{x}, x]$ if $x \geq(2 \cdot 86)^{2}=29584$ and $x \leq 10^{5}$. A direct computation shows that the result holds if $1500 \leq x \leq 29584$.

Theorem 14. The interval $\left[x^{3},(x+1)^{3}\right]$ contains a prime number for $x \geq e^{e^{15}}$ (see Che10).

Under the Riemann Hypothesis, we have that there exists a prime number in the interval $\left[x-\frac{\sqrt{x} \log ^{2} x}{4 \pi}, x\right]$ for $x \geq 599$ (see [Sch76, Theorem 10]).

Proposition 15. There exists a constant $x_{0}$ such that the interval $\left[x-x^{\frac{2}{3}+0.001}, x\right]$ contains at least two prime numbers when $x \geq x_{0}$. The (best) constant $x_{0}$ is at most $\left(e^{e^{15}}+2\right)^{3}$. Under the Riemann Hypothesis, the (best) constant $x_{0}$ is less than 100 .

Proof. Let us prove the first statement. By Theorem [14, the interval $\left[x^{3},(x+2)^{3}\right]$ contains at least two prime numbers for $x \geq e^{e^{15}}$. Let $y=(x+2)^{3}$. We have that the interval $\left[y-\left(6 \sqrt[3]{y^{2}}-12 \sqrt[3]{y}+8\right), y\right]$ contains at least two prime numbers for $y \geq\left(e^{e^{15}}+2\right)^{3}$. Now, we have that $\left[y-\left(6 \sqrt[3]{y^{2}}-12 \sqrt[3]{y}+8\right), y\right] \subseteq\left[y-y^{\frac{2}{3}+0.001}, y\right]$, hence $\left[y-y^{\frac{2}{3}+0.001}, y\right]$ contains at least two prime numbers for $y \geq\left(e^{e^{15}}+2\right)^{3}$.

Assume the Riemann Hypothesis. Let $g(x)=\frac{\sqrt{x} \log ^{2} x}{4 \pi}$. By Theorem 14, $[x-g(x)-g(x-g(x)), x]$ contains at least two prime numbers for $x-g(x) \geq 599$, hence for $x \geq 615$. Since $g(x)+g(x-g(x)) \leq \frac{g(x)}{2}$, we get that there are at least two prime numbers in the interval $\left[x-\frac{\sqrt{x} \log ^{2} x}{4 \pi}, x\right]$ for $x \geq 615$. Since $\frac{\log ^{2} x}{2 \pi} \leq x^{\frac{1}{6}+0.001}$ for $x \geq 4.2 \cdot 10^{16}$, we have that there are at least two primes in $\left[x-x^{\frac{2}{3}+0.001}, x\right]$ for $x \geq 4.2 \cdot 10^{16}$. By Lemma 13 and a direct computation, we have the claim.

Now we are ready to prove the main proposition.

Proposition 16. Assume that $(k, a)$ is an admissible pair. If $k \geq\left(e^{e^{15}}+2\right)^{3}$ or $k \leq 4.2 \cdot 10^{16}$, then $k$ satisfies $(\mathrm{C})$.

Assuming the Riemann Hypothesis, we have that $k$ satisfies (C) for any $k$, i.e. the Riemann Hypothesis implies Conjecture 7. 
Proof. A direct computation shows that the result holds for $k \leq 10000$.

Let $p_{1}$ and $p_{2}$ be as in the beginning of the section. By Proposition 15, we have that there exists a constant $k_{0}$ such that $p_{2} \in\left[k-3-(k-3)^{\frac{2}{3}+0.001}, k-3\right]$ for $k \geq k_{0}$. Thus $a \leq k-p_{2} \leq(k-3)^{\frac{2}{3}+0.001}+3 \leq k^{0.67}$ if $k \geq 10000$. Let $\theta=0.671$ and assume that $a \geq \frac{e^{\frac{2-\theta}{(1-\theta)^{2}}+1.12}}{2-\theta} \approx 5 \cdot 10^{5}$. By Proposition 11 we have that $(k, a)$ satisfies (C). Thus, if $(k, a)$ is an admissible pair, $a \geq 5 \cdot 10^{5}$ and $k \geq \max \left\{k_{0}, 10000\right\}$, then with $(k, a)$ satisfies $(\mathrm{C})$.

By Lemma 13] and the same argument as above, we have that $a \leq k-p_{2} \leq$ $(k-3)^{0.5}+3 \leq k^{0.509}$ for $10000 \leq k \leq 4.2 \cdot 10^{16}$. Let $\theta=0.51$ and assume that $a \geq \frac{e^{\frac{2-\theta}{(1-\theta)^{2}}+1.12}}{2-\theta} \approx 1020$. By Proposition 11 we have that $(k, a)$ satisfies $(\mathrm{C})$. Thus, if $(k, a)$ is an admissible pair, $1020 \leq a \leq\left(4.2 \cdot 10^{16}\right)^{0.51}$ and $k \geq 10000$, then with $(k, a)$ satisfies $(\mathrm{C})$.

Now, let us prove the conjecture for $a \leq 1020$. By Proposition 12, we may assume that $a \geq 14$. For each $a \in\{14, \ldots, 1020\}$, we find the least positive integer $k(a)$ such that $f(k(a), a, h(k(a), a))>0$ and $h(k(a), a) \geq \pi(h(k(a), a)+a)$. By Corollary 10. we have that the admissible pair $(k, a)$ satisfies (C) if $k \geq k(a)$. Recall that since $a \leq k-p_{2}$, we have that $a \leq k^{0.509}$ for $1000 \leq k \leq 4.2 \cdot 10^{16}$. A direct computation shows that $k(a) \leq 10^{6}$ and $a>k(a)^{0.509}$ for $77 \leq a \leq 1020$. Thus if $k \leq k(a)$, we have that $a>k-p_{2}$, hence we get the result for $77 \leq a \leq 1020$. The other cases can be checked directly since $k(a) \leq 10000$ for $14 \leq a \leq 76$.

By Proposition [15] we have that $k_{0} \leq\left(e^{e^{15}}+2\right)^{3}$. Assuming the Riemann Hypothesis, we have that $k_{0}<10000$, hence we obtain the claim.

\section{The irReducibility of the Dirichlet Polynomial}

In this section we assume that $n$ is a fixed positive integer. Let $G$ be a primitive monolithic group with a simple component isomorphic to $\operatorname{Alt}_{k}$ and let $\operatorname{soc}(G) \cong$ $\mathrm{Alt}_{k}^{n}$.

Definition. Let $f(s)$ be a Dirichlet polynomial. We define

$$
m(f(s))=\min \left\{k: a_{k} \neq 0\right\} \quad \text { and } \quad M(f(s))=\max \left\{k: a_{k} \neq 0\right\} .
$$

Proposition 17. Let $f(x)=a+b x^{l}$ be a polynomial in $D[x]$, where $D=$ $\mathbb{Z}\left[x_{1}, \ldots, x_{k}\right]$ and $a, b \in D-\{0\}, l \geq 1$. Assume $f(x)$ is reducible and $\operatorname{GCD}(a, b)=1$. Then there exists a prime divisor $p$ of $l$ such that $a^{2}, b^{2} \in D^{2 p}$.

Proof. This is left to the reader: just apply [Lan02, Chap. VI, Theorem 9.1].

Proposition 18. Let $f(s)$ be a Dirichlet polynomial in $\mathcal{R}^{\prime}$. Let $r$ be a prime number and $l$ a positive integer such that

$$
f(s)=g(s)+r^{l(1-s)} h(s),
$$

with $g^{(r)}(s)=g(s)$ and $h^{(r)}(s)=h(s)$. Assume that

(1) $g(s)$ is irreducible,

(2) $\frac{M(g(s))}{m(g(s))}>\frac{M(h(s))}{m(h(s))}$.

Then $f(s)$ is irreducible.

Proof. Let $m=\operatorname{lcm}\left\{k: a_{k}(f(s)) \neq 0\right\}$, and let $\pi=\mathrm{p}(m)$. Clearly,

$$
\Psi(f(s))=a+b x_{r}^{l}
$$


is an element of $\mathbb{Z}\left[X_{\pi}\right]$ for some $a, b \in \mathbb{Z}\left[X_{\pi-\{r\}}\right]$ and $x_{r}=\Psi\left(r^{1-s}\right)$. We claim that $\operatorname{GCD}(a, b)=1$. Let $c \in \mathbb{Z}\left[X_{\pi-\{r\}}\right]$ such that $c$ divides $\operatorname{GCD}(a, b)$. By (1), the polynomial $a$ is irreducible, so either $c= \pm a$ or $c= \pm 1$. If $c= \pm a$, then $a$ divides $b$, hence $g(s)$ divides $h(s)$. Thus, there exists $k(s) \in \mathbb{Z}\left[X_{\pi-\{r\}}\right]$ such that $h(s)=g(s) k(s)$. In particular, $m(h(s))=m(g(s)) m(k(s))$ and $M(h(s))=$ $M(g(s)) M(k(s))$, so

$$
\frac{M(h(s))}{m(h(s))}=\frac{M(g(s))}{m(g(s))} \frac{M(k(s))}{m(k(s))} \geq \frac{M(g(s))}{m(g(s))},
$$

against (2). So we conclude that $\operatorname{GCD}(a, b)=1$.

Now we prove the claim of the proposition. For a contradiction, assume that $f(s)$ is reducible. Since $\operatorname{GCD}(a, b)=1$, by Proposition 17, we have that there exists a prime divisor $p$ of $l$ such that $a^{2}, b^{2} \in D^{2 p}$. But this is not possible, since $a$ is irreducible. Hence $f(s)$ is irreducible.

Lemma 19. Let $X$ be an almost simple group with socle $\mathrm{Alt}_{k}$. Let $r$ be a prime number such that $k / 2<r<k-2$. If $K$ is a proper subgroup of $X$ containing a Sylow r-subgroup of $X$ and $K S=X$, then $K$ is an intransitive subgroup of $X$.

Proof. Assume that $K$ is a maximal primitive subgroup of $X$. Note that $K$ must contain a cycle of length $r$. By a result of Jordan (see [Jor57, Note C]), we have that $K$ is at least $(k-r+1)$-transitive. By [Jor73, Théorème I], since $k-r+1>2$, we have that $K$ must contain $\mathrm{Alt}_{k-r}$, hence $K$ is an intransitive maximal subgroup of $X$.

It is easy to see that if $K$ is an imprimitive maximal subgroup of $X$, then $r$ divides $|X: K|$.

Proposition 20. Let $X$ be $\mathrm{Sym}_{k}$ or Alt $\mathrm{Al}_{k}$. An intransitive subgroup $N_{\Delta}$ of $X$, which is intersection of maximal subgroups of $X$, is isomorphic to $X \cap \prod_{i=1}^{l} \mathrm{Sym}_{\left|\Delta_{i}\right|}$, where $\Delta=\left\{\Delta_{1}, \ldots, \Delta_{l}\right\}$ be a partition of $\{1, \ldots, k\}$ for some positive integer $l \leq k$. In particular we have that

(1) $\operatorname{Alt}_{k} N_{\Delta}=X$ and

(2) $\mu_{\mathrm{Sym}_{k}}\left(N_{\Delta}\right)=(-1)^{l-1}(l-1)$ !

Proof. This is well known; for the second statement, see [Sta97, p.128].

Let $m$ be a natural number, and let $\Lambda_{m}$ be the set

$$
\left\{\frac{m !}{i_{1} ! \cdots i_{j} !}: \exists j \in \mathbb{N}, i_{1}, \ldots, i_{j} \in \mathbb{N}, i_{1}+\cdots+i_{j}=m\right\} .
$$

Let $1 \leq l \leq \frac{k-1}{2}$. Let $\pi$ be a set of prime numbers satisfying the following:

- $\mathrm{p}(k(k-1) \ldots(k-l+1)) \cap \pi=\varnothing$,

- $\mathrm{p}\left(\begin{array}{l}k \\ i\end{array}\right) \cap \pi \neq \varnothing$ for $l+1 \leq i \leq k / 2$,

- there exists $r \in \pi$ such that $k / 2<r<k-2$.

Let $c$ be an $l+1 \times l$ ! matrix with integer coefficients such that $c_{0,1}=1$ and $c_{m, 1}=-1$ for $m \in\{1, \ldots, l\}$. We define the Dirichlet polynomial (which is an element of $\mathcal{R}^{\prime}$ )

$$
T_{l, c}(s)=\sum_{m=0}^{l} \sum_{i \in \Lambda_{m}} c_{m, i}\left(i\left(\begin{array}{c}
k \\
m
\end{array}\right)\right)^{n(1-s)} .
$$

Clearly, $T_{l, c}(s)$ also depends on $n$, but throughout this section we assume that $n$ is a fixed constant. 
By Lemma 5, Theorem 6, Lemma 19, and Proposition 20, there exists a matrix $c$ such that

$$
P_{G, \operatorname{soc}(G)}^{(\pi)}(s)=T_{l, c}(s) .
$$

Lemma 21. Let $k \geq 6$ and $\mathrm{p}(k) \neq\{2\}$. The Dirichlet polynomial $T_{2, c}(s)$ is irreducible for each $c$.

Proof. Let $h$ and $m$ be natural numbers such that $k=2^{m} h$ and $h>1$ is odd. We get $T_{2, c}(s)=1-g(s) h^{n(1-s)}$, where

$$
g(s)=2^{m n(1-s)}+\left(\frac{2^{m}\left(2^{m} h-1\right)}{2}\right)^{n(1-s)}-c_{2,2}\left(2^{m}\left(2^{m} h-1\right)\right)^{n(1-s)} .
$$

For a contradiction, assume that $T_{2, c}(s)$ is reducible. Since $h>1$ and $\left(2^{m} h-\right.$ $1, h)=1$, by Proposition [17, we have that $g(s)$ is a non-trivial power. This is impossible.

Lemma 22. Let $k \geq 8$. The Dirichlet polynomial $T_{3, c}(s)$ is irreducible for each $c$.

Proof. Let $h$ and $m$ be natural numbers such that $k=2^{m} h$ and $h$ is odd.

Assume that $h>1$. We get $T_{3, c}(s)=1-g(s) h^{n(1-s)}$, where

$$
\begin{aligned}
g(s)= & 2^{m n(1-s)}+\left(\frac{2^{m}(k-1)}{2}\right)^{n(1-s)}+\left(\frac{2^{m}(k-1)(k-2)}{6}\right)^{n(1-s)} \\
& -c_{2,2}\left(2^{m}(k-1)\right)^{n(1-s)}+-c_{3,3}\left(\frac{2^{m}(k-1)(k-2)}{2}\right)^{n(1-s)} \\
& -c_{3,6}\left(2^{m}(k-1)(k-2)\right)^{n(1-s)} .
\end{aligned}
$$

For a contradiction, assume that $T_{3, c}(s)$ is reducible. Since $h>1$ and $((k-1)(k-2), h)=1$, by Proposition 17, we have that $g(s)$ is a non-trivial power. This is impossible.

Assume that $h=1$. We get $f(s)=1-2^{m n(1-s)}+g(s)\left(\frac{k-1}{|k-1|_{3}}\right)^{n(1-s)}$, where

$$
\begin{aligned}
g(s)= & \left(2^{(m-1)}|k-1|_{3}\right)^{n(1-s)}+\left(\frac{2^{m-1}|k-1|_{3}(k-2)}{6}\right)^{n(1-s)} \\
& -c_{2,2}\left(2^{m}|k-1|_{3}\right)^{n(1-s)}+-c_{3,3}\left(2^{m-1}|k-1|_{3}(k-2)\right)^{n(1-s)} \\
& -c_{3,6}\left(2^{m}|k-1|_{3}(k-2)\right)^{n(1-s)} .
\end{aligned}
$$

For a contradiction, assume that $f(s)$ is reducible. Note that $k-1>|k-1|_{3}$, since the equation $k-1=2^{m}-1=3^{l}$ has no solution for $k \geq 5$. Since $\left(3 k(k-2), \frac{k-1}{|k-1|_{3}}\right)=$ 1 and $\left(g(s), 1-2^{m n(1-s)}\right)=1$, by Proposition [18, we have that $g(s)$ is a non-trivial power. This is impossible.

Theorem 23. Let $k$ be an integer such that $(k, a)$ is an admissible pair which satisfies (C) for each a (see the definitions after Conjecture 7). Let $G$ be a primitive monolithic group with a simple component isomorphic to $\operatorname{Alt}_{k}$. Then $P_{G, \operatorname{soc}(G)}(s)$ is irreducible.

Proof. Using the notation of Conjecture [7, for each $a \in\left\{3, \ldots, k-p_{2}+1\right\}$, let $\gamma(k, a)$ be the minimum $h$ such that $(k, a, h)$ satisfies $(\mathrm{C})$.

We construct a finite sequence $\left\{r_{i}\right\}_{1 \leq i \leq \iota}$ of prime numbers and a finite sequence $\left\{a_{i}\right\}_{1 \leq i \leq \iota}$ of positive integers, for some $\iota \in \mathbb{N}-\{0\}$. 
Let $r_{1}$ be a prime number greater than 4 such that $r_{1} \in \mathrm{p}((k-2)(k-3))$ (it is easy to see that such $r_{1}$ exists provided that $\left.k \neq 5,6,11\right)$. In particular, if $k=|k|_{2}$, then take $r_{1} \in \mathrm{p}(k-3)$ such that $r_{1}>4$ (it always exists). Let $a_{1}=j+1$ where $j$ is the unique number such that $j \in\{2,3\}$ and $r_{1}$ divides $k-j$ (in particular, if $|k|_{2}=k$, then $\left.a_{1}=4\right)$. Note that $2<a_{1} \leq 4$, the prime $r_{1}$ does not divide $\frac{k !}{\left(k-a_{1}+1\right) !}$, and $\left|\frac{k !}{\left(k-a_{1}-j_{1}\right) !}\right|_{r_{1}}=\left|\frac{k !}{\left(k-a_{1}-j_{2}\right) !}\right|_{r_{1}}$ for each $0 \leq j_{1}, j_{2} \leq 4$.

Assume that $i \geq 2$. If $a_{i-1}>k-p_{2}$, then we set $\iota=i-1$. If $a_{i-1} \leq$ $k-p_{2}$, then we let $r_{i}$ be a prime number greater than $a_{i-1}+\gamma\left(k, a_{i-1}\right)$ such that $r_{i} \in \mathrm{p}\left(\frac{\left(k-a_{i-1}\right) !}{\left(k-a_{i-1}-\gamma\left(k, a_{i-1}\right)\right) !}\right)$ (it exists since $\left(k, a_{i-1}, \gamma\left(k, a_{i-1}\right)\right)$ satisfies $\left.(\mathrm{C} 2)\right)$. Set $a_{i}=j+1$ where $j$ is the unique number $a_{i-1} \leq j \leq a_{i-1}+\gamma\left(k, a_{i-1}\right)-1$ such that $r_{i}$ divides $k-j$. Note that:

(I) $a_{i-1}<a_{i} \leq a_{i-1}+\gamma\left(k, a_{i-1}\right)$,

(II) the prime $r_{i}$ does not divide $\frac{k !}{\left(k-a_{i}+1\right) !}$,

(III) $\left|\frac{k !}{\left(k-a_{i}-j_{1}\right) !}\right|_{r_{i}}=\left|\frac{k !}{\left(k-a_{i}-j_{2}\right) !}\right|_{r_{i}}$ for each $0 \leq j_{1}, j_{2} \leq a_{i-1}+\gamma\left(k, a_{i-1}\right)$.

Without loss of generality, we may assume that $r_{\iota}=p_{2}$, hence $a_{\iota}=k-p_{2}+1$.

Recall that $k / 2<p_{2}<k-3$. Let $P(s)=P_{G, \operatorname{soc}(G)}(s)$. By Lemma 19] if $M$ is a subgroup of $X$ such that $M$ is intersection of maximal subgroups, $M$ Alt $_{k}=X$ and $|X: M|_{p_{2}}=1$, then $M$ is an intransitive subgroup. In particular, $|X: M|=$ $\frac{k !}{c_{1} ! \ldots l !}$ for some $1 \leq c_{1} \leq c_{2} \leq \cdots \leq c_{l}$ such that $\sum_{i=1}^{l} c_{i}=k$. We claim that $a_{\left(\begin{array}{c}k \\ k-a_{i}\end{array}\right)}(X, S) \neq 0$ for $1 \leq i \leq \iota-1$ and $a_{\frac{k !}{\left(k-a_{i}+1\right) !}}(X, S) \neq 0$ for each $1 \leq i \leq \iota$. Now, let $M$ be a subgroup of index $\frac{k !}{\left(k-a_{i}+1\right) !}$. We claim that $M \cong \operatorname{Sym}_{k-a_{i}+1} \cap X$. As above, we have $\frac{k !}{\left(k-a_{i}+1\right) !}=|X: M|=\frac{k !}{c_{1} ! \ldots c_{l} !}$. Since the prime numbers $r_{i}, \ldots, r_{\iota}$ do not divide $\frac{k !}{\left(k-a_{i}+1\right) !}$, it is easy to see that $c_{l} \geq k-a_{i}+1$ and the claim follows. By Proposition [20, we conclude that $a_{\frac{k !}{\left(k-a_{i}+1\right) !}}(X, S) \neq 0$. Similarly we have that $a_{\left(\begin{array}{c}k \\ k-a_{i}\end{array}\right)}(X, S) \neq 0$.

By the above argument and Theorem [6, we have that

$$
\begin{aligned}
P^{\left(r_{i}, \ldots, r_{\iota}\right)}(s) & =b_{0}(s)+b_{1}(s) r_{1}^{\alpha_{1}(1-s)}+b_{2}(s) r_{2}^{\alpha_{2}(1-s)}+\cdots+b_{i-1}(s) r_{i-1}^{\alpha_{i-1}(1-s)} \\
& =T_{a_{i}-1, c_{i}}(s)
\end{aligned}
$$

and $P^{\left(r_{1}, \ldots, r_{\iota}\right)}(s)=b_{0}(s)=T_{a_{1}-1, c}(s)$, for some $\alpha_{i} \in \mathbb{Z}$, some matrices $c_{i}$ and $c$, and some $b_{i}(s) \in \mathcal{R}$ such that $b_{i-1}^{\left(r_{i-1}\right)}(s)=b_{i-1}(s)$ for $2 \leq i \leq \iota$. Moreover, $m\left(b_{i}(s) r_{i}^{\alpha_{i}(1-s)}\right)=\left(\begin{array}{c}k \\ k-a_{i}\end{array}\right)^{n}$ and $M\left(b_{i}(s) r_{i}^{\alpha_{i}(1-s)}\right)=\left(\frac{k !}{\left(k-a_{i+1}+1\right) !}\right)^{n}$ for $1 \leq i \leq \iota-1$, $m\left(b_{0}(s)\right)=1, M\left(b_{0}(s)\right)=\left(\frac{k !}{\left(k-a_{1}+1\right) !}\right)^{n}$.

Note that by Lemma 21 and Lemma 22, we have that $b_{0}(s)$ is irreducible (in fact, by Lemma [5. Lemma 19] and Proposition 20. if $a_{1}=3$, then $b_{0}(s)=f(s)$ in Lemma 21, if $a_{1}=4$, then $b_{0}(s)=f(s)$ in Lemma 22, moreover note that if $|k|_{2}=k$, then $\left.a_{1}=4\right)$. We claim that $P^{\left(r_{i}, \ldots, r_{\iota}\right)}(s)$ is irreducible for each $1 \leq i \leq \iota$. We argue by induction. For $i=1$ the result is clear, since $P^{\left(r_{1}, \ldots, r_{\iota}\right)}(s)=b_{0}(s)$.

Now we assume that $P^{\left(r_{i}, \ldots, r_{\iota}\right)}(s)$ is irreducible for some $i \in\{1, \ldots, \iota-1\}$ and we claim that $P^{\left(r_{i+1}, \ldots, r_{\iota}\right)}(s)$ is irreducible. We have that

$$
P^{\left(r_{i+1}, \ldots, r_{\iota}\right)}(s)=P^{\left(r_{i}, \ldots, r_{\iota}\right)}(s)+b_{i}(s) r_{i}^{\alpha_{i}(1-s)} .
$$


We apply Proposition 18. Clearly, $b_{i}^{\left(r_{i}\right)}(s)=b_{i}(s)$ and $P^{\left(r_{i}, r_{i}, \ldots, r_{\iota}\right)}(s)=P^{\left(r_{i}, \ldots, r_{\iota}\right)}(s)$. Moreover, $P^{\left(r_{i}, \ldots, r_{\iota}\right)}(s)$ is irreducible by inductions, so condition (1) is satisfied. Now, we check that condition (2) is satisfied. Since $\left(k, a_{i}, \gamma\left(k, a_{i}\right)\right)$ satisfies $(\mathrm{C} 1)$, we have that

$$
\left(\begin{array}{c}
k \\
a_{i}
\end{array}\right)>\frac{\left(k-a_{i}+1\right) !}{\left(k-a_{i}-\gamma\left(k, a_{i}\right)+1\right) !} .
$$

Since $a_{i+1} \leq a_{i}+\gamma\left(k, a_{i}\right)$ (see (I) above), we obtain

$$
\left(\begin{array}{c}
k \\
a_{i}
\end{array}\right)>\frac{\left(k-a_{i}+1\right) !}{\left(k-a_{i+1}+1\right) !}
$$

which is equivalent to $\frac{M\left(P^{\left(r_{i}, \ldots, r_{\iota}\right)}(s)\right)}{m\left(P^{\left(r_{i}, \ldots, r_{\iota}\right)}(s)\right)}>\frac{M\left(b_{i+1}(s) r_{i+1}^{\alpha_{i+1}(1-s)}\right)}{m\left(b_{i+1}(s) r_{i+1}^{\alpha_{i+1}(1-s)}\right)}=\frac{M\left(b_{i+1}(s)\right)}{m\left(b_{i+1}(s)\right)}$. Thus condition (2) of Proposition 18 holds. Hence we conclude that $P^{\left(r_{i}, \ldots, r_{\iota}\right)}(s)$ is irreducible.

In particular, we have that $P^{\left(r_{\iota}\right)}(s)=P^{\left(p_{2}\right)}(s)$ is irreducible.

Now, assume that $P(s)=f(s) g(s)$ for some Dirichlet polynomials $f(s)$ and $g(s)$. Since $P^{\left(p_{2}\right)}(s)=f^{\left(p_{2}\right)}(s) g^{\left(p_{2}\right)}(s)$ is irreducible, without loss of generality, we may assume that $P^{\left(p_{2}\right)}(s)=f^{\left(p_{2}\right)}(s)$ and $g^{\left(p_{2}\right)}(s)=1$. Now, $\left|P^{\left(p_{2}\right)}(s)\right|_{p_{1}}=|P(s)|_{p_{1}}$, hence $|f(s)|_{p_{1}}=|P(s)|_{p_{1}}$ and $|g(s)|_{p_{1}}=1$. It follows that $g^{\left(p_{1}\right)}(s)=g(s)$. By Lemma 19, we have that $P^{\left(p_{1}\right)}(s)=P^{\left(p_{1}, p_{2}\right)}(s)$. Thus $g^{\left(p_{1}\right)}(s)=g^{\left(p_{1}, p_{2}\right)}(s)=1$, so $g(s)=1$.

Theorem 24. Let $5 \leq k \leq 15$ or $k \in\{19,20,21,55,84\}$. Let $G$ be a primitive monolithic group with a simple component isomorphic to $\mathrm{Alt}_{k}$. Moreover, if $k=$ 6 , then assume that $N_{G}(s) / C_{G}(s)$ is isomorphic to a subgroup of $\mathrm{Sym}_{6}$. Then $P_{G, \operatorname{soc}(G)}(s)$ is irreducible.

Proof. We let $P(s)=P_{G, \operatorname{soc}(G)}(s)$. Assume that $P(s)=f(s) g(s)$.

TABLE 1. Some cases

\begin{tabular}{|c|c|c|}
\hline$k$ & $r$ & $\pi$ \\
\hline 5 & 3 & $\{2,5\}$ \\
6 & 2 & $\{3,5\}$ \\
7 & 3 & $\{5,7\}$ \\
10 & 2 & $\{5,7\}$ \\
11 & 5 & $\{7,11\}$ \\
12 & 2 & $\{3,11\}$ \\
\hline
\end{tabular}

Case $k=5,6,7,10,11,12$. Let $r$ and $\pi$ be as in Table 1. Using GAP, we have that $P^{(r)}(s)$ is irreducible. So we may assume that $f^{(r)}(s)=P^{(r)}(s)$ and $g^{(r)}(s)=1$. Note that $\left|P^{(r)}(s)\right|_{p}=|P(s)|_{r}$ for $p \in \pi$, hence $|f(s)|_{p}=|P(s)|_{p},|g(s)|_{p}=1$ and $g^{(p)}(s)=g(s)$ for $p \in \pi$. In particular, $g^{(\pi)}(s)=g(s)$, but $P^{(\pi)}(s)=1$. This concludes the proof.

Case $k=8$. Using GAP, we have that $P^{(2)}(s)$ and $P^{(5)}(s)$ are irreducible. So we may assume that $f^{(2)}(s)=P^{(2)}(s)$ and $g^{(2)}(s)=1$. Note that $\left|P^{(2)}(s)\right|_{r}=|P(s)|_{r}$ for $r \in\{3,5\}$, hence $|f(s)|_{r}=|P(s)|_{r},|g(s)|_{r}=1$ and $g^{(r)}(s)=g(s)$ for $r \in\{3,5\}$. 
Since $\left|P^{(5)}(s)\right|_{3}>1$ and $P^{(5)}(s)$ is irreducible, we have that $P^{(5)}(s)=f^{(5)}(s)$. Thus $g^{(5)}(s)=1$ and so $g(s)=g^{(5)}(s)=1$. This concludes the proof.

Case $k=9$. Using GAP, we have that $P^{(5)}(s)$ is irreducible. So we may assume that $f^{(5)}(s)=P^{(5)}(s)$ and $g^{(5)}(s)=1$. Note that $\left|P^{(5)}(s)\right|_{7}=|P(s)|_{7}$, hence $|f(s)|_{7}=|P(s)|_{7},|g(s)|_{7}=1$. Since

$$
P^{(3)}(s)=1-280^{n(1-s)},
$$

and $|g(s)|_{7}=1$ we have that $f^{(3)}(s)=P^{(3)}(s)$. In particular, $\left|P^{(3)}(s)\right|_{5}=|P(s)|_{5}$, hence $|f(s)|_{5}=|P(s)|_{5}$ and $|g(s)|_{5}=1$. So $g(s)=g^{(5)}(s)=1$. This concludes the proof.

Case $k=13,14$. By Lemma 21 and 22 we have that $P^{(11)}(s)$ is irreducible. So we may assume that $f^{(11)}(s)=P^{(11)}(s)$ and $g^{(11)}(s)=1$. Now, $|P(s)|_{13}=\left|P^{(11)}(s)\right|_{13}$, hence $|f(s)|_{13}=|P(s)|_{13}$, thus $|g(s)|_{13}=1$. By Lemma 19 for $k=13$ and by LPS90, Theorem p. 130] we have that $P^{(7,13)}(s)=1$ and $\left|P^{(7)}(s)\right|_{11}=|P(s)|_{11}$. Since $g^{(7)}(s)$ divides $P^{(7)}(s)$ and $|g(s)|_{13}=1$, we have that $g^{(7)}(s)=1$. This implies that $f^{(7)}(s)=P^{(7)}(s)$, hence $|P(s)|_{11}=\left|P^{(7)}(s)\right|_{11}=|f(s)|_{11}$. Hence we obtain $|g(s)|_{11}=1$, so $g^{(11)}(s)=g(s)$. But $g^{(11)}(s)=1$, thus $g(s)=1$ and we get the claim.

Case $k=15,19,21$. Let $\left(p_{1}, p_{2}\right)=(13,11)$ if case $k=15$ holds, $\left(p_{1}, p_{2}\right)=(19,17)$ otherwise. By Lemma 22 we have that $P^{\left(p_{2}\right)}(s)$ is irreducible for $k=19$. Arguing as in Lemma 22 we have that $P^{\left(p_{2}\right)}(s)=T_{4}(s)$ is irreducible for $k=15,21$. So we may assume that $f^{\left(p_{2}\right)}(s)=P^{\left(p_{2}\right)}(s)$ and $g^{\left(p_{2}\right)}(s)=1$. Now, $|P(s)|_{p_{1}}=\left|P^{\left(p_{2}\right)}(s)\right|_{p_{1}}$, hence $|f(s)|_{p_{1}}=|P(s)|_{p_{1}}$, thus $|g(s)|_{p_{1}}=1$ and $g^{\left(p_{1}\right)}(s)=g(s)$. But $P^{\left(p_{1}\right)}(s)=$ $P^{\left(p_{2}, p_{1}\right)}(s)=f^{\left(p_{1}\right)}(s)$, hence $g^{\left(p_{1}\right)}(s)=1$. So $g(s)=g^{\left(p_{1}\right)}(s)=1$ and we get the claim.

Case $k=55$. By Lemma 22 the Dirichlet polynomial $P^{(13,47)}(s)$ is irreducible. So we may assume that $P^{(13,47)}(s)=f^{(13,47)}(s)$. Moreover we have $|P(s)|_{53}=$ $\left|P^{(13,47)}(s)\right|_{53}$, hence $|f(s)|_{53}=|P(s)|_{53}$, so $|g(s)|_{53}=1$ and $g^{(53)}(s)=g(s)$. Now, $P^{(53)}(s)=P^{(53,13,47)}(s)$, hence $f^{(53)}(s)=P^{(53)}(s)$ and $g^{(53)}(s)=1$. This proves the claim.

Case $k=20,84$. Let $\left(\pi, p_{1}, p_{2}, p_{3}\right)=(\{17\}, 19,13,17)$ if case $k=20$ holds, $\left(\pi, p_{1}, p_{2}, p_{3}\right)=(\{5,79\}, 83,73,79)$ otherwise. By Lemma 22 the Dirichlet polynomial $P^{(\pi)}(s)$ is irreducible. So we may assume that $P^{(\pi)}(s)=f^{(\pi)}(s)$. Moreover we have $|P(s)|_{p_{1}}=\left|P^{(\pi)}(s)\right|_{p_{1}}$, hence $|f(s)|_{p_{1}}=|P(s)|_{p_{1}}$, so $|g(s)|_{p_{1}}=1$ and $g^{\left(p_{1}\right)}(s)=g(s)$. Now, by [Mas07, Lemma 2] we have that

$$
P^{\left(p_{1}\right)}(s) \text { divides }\left(1-k^{n(1-s)}\right)\left(1-((k-3) !)^{n(1-s)}\right) .
$$

Let $h(s)=\operatorname{GCD}\left(g^{\left(p_{1}\right)}(s), 1-k^{n(1-s)}\right)$. It is easy to see that

$$
h^{(\pi)}(s)=\operatorname{GCD}\left(g^{\left(\pi \cup\left\{p_{1}\right\}\right)}(s), 1-k^{n(1-s)}\right)=1
$$

since $g^{(\pi)}(s)=1$. Thus $g(s)=g^{\left(p_{1}\right)}(s)$ must divide $1-((k-3) !)^{n(1-s)}$. This implies that $P^{\left(p_{2}\right)}(s)=f^{\left(p_{2}\right)}(s)$, but $\left|P^{\left(p_{2}\right)}(s)\right|_{p_{3}}=|P(s)|_{p_{3}}$, hence $|f(s)|_{p_{3}}=|P(s)|_{p_{3}}$ and $|g(s)|_{p_{3}}=1$. Since $g(s)$ divides $1-((k-3) !)^{n(1-s)}$, it must be $g(s)=1$. 


\section{RECOGNIZING THE NUMBER OF FACTORS OF THE SOCLE OF $L$ FROM $P_{L, N}(s)$}

In this section let $L$ be a monolithic primitive group with non-abelian socle $N$ isomorphic to a direct product of $n$ copies of a simple group $S$. Our aim is to recognize the number $n$ from the knowledge of the Dirichlet polynomials $\left\{P_{L, N}^{(r)}(s)\right.$ : $r \in p(S)\}$ (see Proposition 27).

Lemma 25. Let $p$ be a prime number, and let $m$ be a positive integer.

(1) If $p^{m}+1$ is a non-trivial power of an integer, then $(p, m)=(2,3)$ or $(p, m)=\left(2^{k}-1,1\right)$ for some $k \in \mathbb{N}$.

(2) If $p \not \equiv 2(\bmod 3)$, then $p^{2 m}+p^{m}+1$ is not a non-trivial power.

(3) If $p=2^{k}-1$ and $k \geq 5$, then $\frac{p^{2}+1}{2}$ is not a non-trivial power.

Proof. Let us prove (1). Assume that $p^{m}+1=a^{l}$ for some positive integers $a$ and $l$, such that $l \geq 2$. Assume that $a=2$. It is easy to see that in this case $p^{m}=p$ is a Mersenne prime. Assume that $a>2$. We have that $p^{m}=a^{l}-1$ and $p$ divides $a-1$, hence there are no Zsigmondy primes for $\langle a, l\rangle$. The claim follows.

Now we prove (2). Assume that $p^{2 m}+p^{m}+1=a^{l}$ for some positive integers $a$ and $l$, such that $l \geq 2$. Without loss of generality, we may assume that $l$ is a prime number. Suppose that $p=3$. We have that $3^{m}\left(3^{m}+1\right)=a^{l}-1$. Clearly 3 does not divide $a$. Assume that $a \equiv 2(\bmod 3)$. In this case $l$ must be even, hence $l=2$. Therefore $3^{m}$ divides $a-1$ or $a+1$, which are even numbers, hence $a \geq 2 \cdot 3^{m}-1$. So $3^{2 m}+3^{m}+1=a^{2} \geq 4 \cdot 3^{2 m}-4 \cdot 3^{m}+1$, that implies $m \leq 0$, a contradiction. Assume that $a \equiv 1(\bmod 3)$. Suppose that 3 does not divide $l$. Since $\left(a-1, a^{l-1}+\cdots+1\right)$ divides $l$, we have that $3^{m}$ divides $a-1$, hence $a \geq 2 \cdot 3^{m}+1$, which is impossible. So we have that $l=3$. In this case $3^{m}\left(3^{m}+1\right)=(a-1)\left(a^{2}+a+1\right)$. Note that $\left|a^{2}+a+1\right|_{3}=3$ since $a^{2}+a+1 \equiv 3(\bmod 9)$ when $a \equiv 1(\bmod 3)$. Hence $2 \cdot 3^{m-1}$ divides $a-1$, so $a \geq 2 \cdot 3^{m-1}+1$. It follows that $3^{2 m}+3^{m}+1=a^{3} \geq\left(2 \cdot 3^{m-1}+1\right)^{3}$ which does not occur. Finally, suppose that $p \neq 3$. In this case, we have that $p \equiv 1$ $(\bmod 3)$, hence $\left|p^{2}+p+1\right|_{3}=3$, as we have seen above. This implies that $l=1$.

Finally we prove (3). Assume that $p^{2}+1=2 a^{l}$ for some positive integers $a$ and $l$, such that $l \geq 2$. Without loss of generality, we may assume that $l$ is a prime number. We have that $2^{k}\left(2^{k-1}-1\right)=a^{l}-1$. If $l$ is odd, then $2^{k}$ divides $a-1$, hence $a \geq 2^{k}+1$ and we get a contradiction. If $l=2$, then $2^{k-1}$ divides $a-1$ or $a+1$. It is easy to see that if $a-1$ or $a+1$ equals $2^{k-1}$, then we have a contradiction. Hence $a \geq 3 \cdot 2^{k-1}-1$, so $2^{2 k-1}-2^{k}+1=a^{2} \geq 9 \cdot 2^{2 k-2}-6 \cdot 2^{k-1}+1$, which does not occur.

We recall a useful result.

Theorem 26 ([DL07, Theorem 1.1]). Let $X$ be an almost simple group with socle $S$. For any $p \in \mathrm{p}(S)$, there exists a positive integer $m$ with the following properties:

- $p$ divides $m$;

- there exists a prime which divides $|S|$ but does not divide m;

- $a_{m}(X, S) \neq 0$.

Proposition 27. Let $L$ be a monolithic primitive group with socle $N \cong S^{n}$, where $S$ is a non-abelian simple group and $n=\left|L: N_{L}(S)\right|$. Then

$$
n=\max \left\{a \in \mathbb{N}: \forall k \in \mathbb{N}\left(\text { if } a_{k}(L, N) \neq 0 \text { and } \mathrm{p}(k) \neq \mathrm{p}(S) \text {, then } k \in \mathbb{Z}^{a}\right)\right\} .
$$



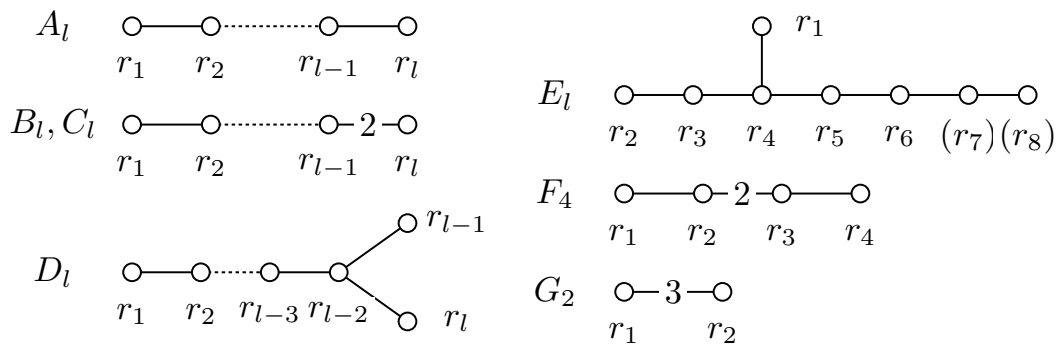

Figure 1. Dynkin diagrams

So, if we know the Dirichlet polynomials $\left\{P_{L, N}^{(r)}(s): r \in \mathrm{p}(S)\right\}$, then $n$ is uniquely determined.

Proof. By Theorem 6] if $a_{k}(L, N) \neq 0$ and $\mathrm{p}(k) \neq \mathrm{p}(S)$, then $k=l^{n}$ and $a_{l}(X, S) \neq$ 0 , where $X=N_{L}(S) / C_{L}(S)$. So it suffices to prove that if $X$ is an almost simple group with socle $S$, then there exists a positive integer $m$ such that $m$ is not a non-trivial power, $a_{m}(X, S) \neq 0$ and $\mathrm{p}(m) \neq \mathrm{p}(S)$.

Assume that there exists a prime number $r$ such that $|S|_{r}=r$. By Theorem 26 , we have that there exists a positive integer $m$ such that $r$ divides $m, \mathrm{p}(m) \neq \mathrm{p}(S)$ and $a_{m}(X, S) \neq 0$. In particular, $m$ is not a non-trivial power. Thus we have the claim.

Let $m_{1}$ and $m_{2}$ be two integers such that $m_{2}$ divide $m_{1}, \mathrm{p}\left(m_{i}\right) \neq \mathrm{p}(S)$ and $a_{m_{i}}(X, S) \neq 0$. Note that to obtain the claim, it suffices to show that $\frac{m_{1}}{m_{2}}$ is not a non-trivial power. So, in order to prove our proposition, we show that

(a) there exists a prime number $r$ such that $|S|_{r}=r$, or

(b) there exist $k \geq 1$ and some subgroups $H, H_{1}, \ldots, H_{k}$ of $X$ such that $X=$ $H S, a_{\left|X: H_{i}\right|}(X, S) \neq 0, a_{|X: H|}(X, S) \neq 0, \mathrm{p}(|X: H|) \neq \mathrm{p}(S)$ and $\frac{|X: H|}{\left|X: H_{i}\right|}$ is not a non-trivial power of an integer for some $i \in\{1, \ldots, k\}$.

If $S$ is sporadic, then (a) occurs. Moreover, if $S$ is alternating then by the Bertrand postulate there exists a prime number in the open interval $(n / 2, n)$ for $n \geq 5$. So (a) occurs.

From now on, assume that $S$ is a group of Lie type over a field of characteristic $p$. Let $B$ be a Borel subgroup of $S$. Let $\rho$ be the symmetry of the Dynkin diagram which defines $S$ : if $S$ is untwisted, then $\rho$ is trivial, otherwise $\rho$ has order 2 or 3. In Table 2, given a simple group of Lie type $S$, we describe two $\rho$-invariant sets of roots $K_{1}$ and $K_{2}$, referring to Figure 1] In the second column we give some conditions, in particular referring to the graph automorphisms contained in $X$ : when we write no graph, we mean that $X$ does not contain a non-trivial graph automorphism; when we write graph, we mean that $X$ contains a non-trivial graph automorphism. Sometimes we specify the order of the graph automorphism (for instance, we write 3-graph in the case when $S=D_{4}(q)$ and $X$ contains a graph automorphism of order 3). If $P$ is a parabolic subgroup of $S$, we denote by $L_{P}$ the Levi factor of $P$. For $i \in\{1,2\}$, we let $K_{i}^{\prime}$ be the set of the $\rho$-orbits which are in $K_{i}$. Using the notation of [Pat09, Section 2], we associate to each set of $\rho$-orbits $K_{i}^{\prime}$ a parabolic subgroup $P_{K_{i}^{\prime}}$ of $S$. Now, let $K_{i}$ be as in Table 2] We have that $N_{X}\left(P_{K_{i}^{\prime}}\right)$ is subgroup of $X$ such that $N_{X}\left(P_{K_{i}^{\prime}}\right) S=X$, and if $Q$ is another subgroup of $X$ such that $|X: Q|=\left|X: N_{X}\left(P_{K_{i}^{\prime}}\right)\right|$ and $Q S=X$, then $Q \cap S$ and $P_{K_{i}^{\prime}}$ have the same 
Levi factor $L_{P_{K_{i}^{\prime}}}$. By [Pat09, Theorem 17], we conclude that $a_{\left|S: P_{K_{i}^{\prime}}\right|}(X, S) \neq 0$ and $a_{|S: B|}(X, S) \neq 0$. Moreover, $p$ does not divide $|S: B|$.

We want to prove that (b) holds whenever (a) does not hold. Let $H=N_{X}(B)$ and $H_{i}=N_{X}\left(P_{K_{i}^{\prime}}\right)$. Note that it remains to prove that $\frac{|X: H|}{\left|X: H_{i}\right|}=\frac{|S: B|}{\left|S: P_{K_{i}^{\prime}}\right|}$ is not a non-trivial power for some $i \in\{1,2\}$, whenever (a) does not hold. In Table 3, we report the index $|P: B|$, where $P$ is a parabolic subgroup, given the Levi factor $L_{P}$ (see [Pat09, Section 3]).

TABLE 2. Some Levi factors of a simple group of Lie type.

\begin{tabular}{|c|c|c|c|c|c|}
\hline$S$ & Conditions & $K_{1}$ & $L_{P_{K_{1}^{\prime}}}$ & $K_{2}$ & $L_{P_{K_{2}^{\prime}}}$ \\
\hline$A_{1}(q)$ & & $\left\{r_{1}\right\}$ & $A_{1}(q)$ & & \\
$A_{l}(q)$ & $l \geq 2$, no graph & $\left\{r_{1}, r_{2}\right\}$ & $A_{2}(q)$ & & \\
$A_{l}(q)$ & $l$ even, $l \geq 2$, graph & $\left\{r_{\frac{l}{2}}, r_{\frac{l+2}{2}}\right\}$ & $A_{2}(q)$ & & \\
$A_{l}(q)$ & $l$ odd, $l \geq 3$, graph & $\left\{r_{\frac{l+1}{2}}\right\}$ & $A_{1}(q)$ & $\left\{r_{\frac{l-1}{2}}, r_{\frac{l+1}{2}}, r_{\frac{l+3}{2}}\right\}$ & $A_{3}(q)$ \\
$B_{l}(q), C_{l}(q)$ & $l \geq 3$ & $\left\{r_{1}, r_{2}\right\}$ & $A_{2}(q)$ & & \\
$C_{2}(q)$ & No graph & $\left\{r_{1}\right\}$ & $A_{1}(q)$ & $\left\{r_{1}, r_{2}\right\}$ & $C_{2}(q)$ \\
$C_{2}(q)$ & $q$ even, graph & $\left\{r_{1}, r_{2}\right\}$ & $C_{2}(q)$ & & \\
$D_{l}(q)$ & $l \geq 4$, no 3-graph & $\left\{r_{1}, r_{2}\right\}$ & $A_{2}(q)$ & & \\
$D_{4}(q)$ & 3 -graph & $\left\{r_{2}\right\}$ & $A_{1}(q)$ & $\left\{r_{1}, r_{2}, r_{3}, r_{4}\right\}$ & $D_{4}(q)$ \\
$E_{l}(q)$ & $l \in\{6,7,8\}$ & $\left\{r_{1}, r_{4}\right\}$ & $A_{2}(q)$ & & \\
$F_{4}(q)$ & No graph & $\left\{r_{1}, r_{2}\right\}$ & $A_{2}(q)$ & & \\
$F_{4}(q)$ & $q$ even, graph & $\left\{r_{2}, r_{3}\right\}$ & $C_{2}(q)$ & & \\
$G_{2}(q)$ & No graph & $\left\{r_{1}\right\}$ & $A_{1}(q)$ & $\left\{r_{1}, r_{2}\right\}$ & $G_{2}(q)$ \\
$G_{2}(q)$ & $p=3$, graph & $\left\{r_{1}, r_{2}\right\}$ & $G_{2}(q)$ & & \\
${ }^{2} A_{l}(q)$ & & $\left\{r_{1}, r_{l}\right\}$ & $A_{1}\left(q^{2}\right)$ & & \\
${ }^{2} B_{2}(q)$ & & $\left\{r_{1}, r_{2}\right\}$ & $2 B_{2}(q)$ & & \\
${ }^{2} D_{l}(q)$ & & $\left\{r_{1}, r_{2}\right\}$ & $A_{2}(q)$ & & \\
${ }^{3} D_{4}(q)$ & & $\left\{r_{1}, r_{3}, r_{4}\right\}$ & $A_{1}\left(q^{3}\right)$ & & \\
${ }^{2} E_{6}(q)$ & & $\left\{r_{1}, r_{4}\right\}$ & $A_{2}(q)$ & & \\
${ }^{2} F_{4}(q)$ & & $\left\{r_{1}, r_{4}\right\}$ & $A_{1}\left(q^{2}\right)$ & & \\
${ }^{2} G_{2}(q)$ & & $\left\{r_{1}, r_{2}\right\}$ & $2 G_{2}(q)$ & & \\
\hline
\end{tabular}

In the sequel we repeatedly use Lemma 25 to show that a certain expression is not a non-trivial power.

Assume that $L_{P_{K_{1}}}=A_{2}(q)$. Since $(q+1)\left(q^{2}+q+1\right)=\left|P_{K_{1}}: B\right|=\frac{|S: B|}{\left|S: P_{K_{1}}\right|}$ is not a non-trivial power, we have the claim.

Assume that $L_{P_{K_{1}}}=A_{1}\left(q^{2}\right)$ or ${ }^{2} B_{2}(q)$. Since $q^{2}+1=\left|P_{K_{1}}: B\right|=\frac{|S: B|}{\left|S: P_{K_{1}}\right|}$ is not a non-trivial power, we have the claim.

Assume that $L_{P_{K_{1}}}={ }^{2} G_{2}(q)$ for $p=3$. Since $q^{3}+1=\left|P_{K_{1}}: B\right|=\frac{|S: B|}{\left|S: P_{K_{1}}\right|}$ is not a non-trivial power, we have the claim.

Assume that $S={ }^{3} D_{4}(q)$, so $L_{P_{K_{1}}}=A_{1}\left(q^{3}\right)$. If $q^{3}+1=\left|P_{K_{1}}: B\right|=\frac{|S: B|}{\left|S: P_{K_{1}}\right|}$ is a non-trivial power, then $q=2$. In this case, $\left|{ }^{3} D_{4}(2)\right|_{13}=13$, hence we have the claim. 
TABLE 3. Index of the Borel subgroup in some parabolic subgroups $P$

\begin{tabular}{|c|c|}
\hline$L_{P}$ & $|P: B|$ \\
\hline$A_{1}(q)$ & $q+1$ \\
$A_{1}\left(q^{2}\right)$ & $q^{2}+1$ \\
$A_{1}\left(q^{3}\right)$ & $q^{3}+1$ \\
$A_{2}(q)$ & $\left(q^{2}+q+1\right)(q+1)$ \\
$A_{3}(q)$ & $\left(q^{2}+1\right)\left(q^{2}+q+1\right)(q+1)^{2}$ \\
${ }^{2} B_{2}(q)$ & $q^{2}+1$ \\
$C_{2}(q)$ & $\left(q^{2}+1\right)(q+1)^{2}$ \\
$D_{4}(q)$ & $(q+1)^{4}\left(q^{2}+1\right)^{2}\left(q^{2}+q+1\right)\left(q^{2}-q+1\right)$ \\
${ }^{3} D_{4}(q)$ & $(q+1)^{2}\left(q^{2}-q+1\right)\left(q^{8}+q^{4}+1\right)$ \\
$F_{4}(q)$ & $\left(q^{12}+1\right)\left(q^{8}+1\right)\left(q^{2}-q+1\right)\left(q^{2}+q+1\right)$ \\
$G_{2}(q)$ & $(q+1)^{2}\left(q^{2}+q+1\right)\left(q^{2}-q+1\right)$ \\
${ }^{2} G_{2}(q)$ & $q^{3}+1$ \\
\hline
\end{tabular}

Assume that $L_{P_{K_{1}}}=A_{1}(q)$. If $q+1=\left|P_{K_{1}}: B\right|=\frac{|S: B|}{\left|S: P_{K_{1}}\right|}$ is a non-trivial power, then $q=8$ or $q=2^{k}-1$. So, assume that $q=8$ or $q=2^{k}-1$. We consider some cases.

- Let $S \cong A_{1}(q)$. There exists a prime number $r$ such that $\left|A_{1}(q)\right|_{r}=r$.

- Let $S \cong C_{2}(q)$ and assume that $X$ does not contain a non-trivial graph automorphism. We have that $\left(q^{2}+1\right)(q+1)^{2}=\left|P_{K_{2}}: B\right|=\frac{|S: B|}{\left|S: P_{K_{2}}\right|}$ is not a non-trivial power when $q=8$ (by direct computation) and when $q=2^{k}-1$.

- In the remaining cases, we have that $\left|P_{K_{2}}: B\right|=\frac{|S: B|}{\left|S: P_{K_{2}}\right|}$ is not a non-trivial power when $q \in\left\{8,2^{k}-1\right\}$, since $q^{2}+q+1$ is not a non-trivial power.

Assume that $L_{P_{K_{1}}}=C_{2}(q), q$ is even and $X$ contains a non-trivial graph automorphism. The number $(q+1)^{2}\left(q^{2}+1\right)=\left|P_{K_{1}}: B\right|=\frac{|S: B|}{\left|S: P_{K_{1}}\right|}$ is not a non-trivial power since $q^{2}+1$ is a non-trivial power.

Assume that $S=G_{2}(q), p=3$ and $X$ contains a non-trivial graph automorphism. The number $(q+1)^{2}\left(q^{2}+q+1\right)\left(q^{2}-q+1\right)=\left|P_{K_{1}}: B\right|=\frac{|S: B|}{\left|S: P_{K_{1}}\right|}$ is not a non-trivial power.

\section{ReCognizing the Chief FaCtors From the Dirichlet POlynomial}

Proposition 28. Let $k \geq 5$ and assume that $X$ is an almost simple group with socle $\mathrm{Alt}_{k}$. Let $Z$ be an almost simple group such that $P_{X, \operatorname{Alt}_{k}}^{(r)}(s)=P_{Z, \operatorname{soc}(Z)}^{(r)}(s)$ for each $r \in \mathrm{p}(k !)$. Then $\operatorname{soc}(Z) \cong \operatorname{Alt}_{k}$.

Proof. Let $m$ be the minimal index of a proper subgroup of $X$ which is supplemented by $\mathrm{Alt}_{k}$. We have that $m=10$ if $X \not \leq \mathrm{Sym}_{6}$ and $k=6$, otherwise $m=k$. Let $\pi=\mathrm{p}(k !)-\mathrm{p}(m)$ and note that $\pi \neq \varnothing$. We get $P_{X, \operatorname{Alt}_{k}}^{(\pi)}(s)=P_{Z, \operatorname{soc}(Z)}^{(\pi)}(s)$ hence $a_{m}\left(X, \operatorname{Alt}_{k}\right)=a_{m}(Z, \operatorname{soc}(Z)) \neq 0$. So $m$ is the minimal index of a proper subgroup of $Z$. Moreover, since $Z$ is an almost simple group, we have that $Z$ is a primitive group of degree $m$. 
Let $k=6$ and assume that $X \not \leq \operatorname{Sym}_{6}$. By Theorem $[26$ we have that $\{2,3,5\} \subseteq$ $\mathrm{p}(Z)$. By GAP, if $Z$ is a primitive group of degree $k$ such that $\{2,3,5\} \subseteq \mathrm{p}(Z)$ and the minimal index of a proper subgroup is 10 , then $\operatorname{soc}(Z) \cong \operatorname{Alt}_{6}$.

Assume that if $k=6$, then $X \leq \operatorname{Sym}_{6}$. Let $k \in\{5,6,7\}$. By Theorem 26] we have that $\mathrm{p}(k !) \subseteq \mathrm{p}(Z)$. By GAP, if $Z$ is a primitive group of degree $k$ such that $\mathrm{p}(k !) \subseteq \mathrm{p}(Z)$ and the minimal index of a proper subgroup is $k$, then $\operatorname{soc}(Z) \cong \operatorname{Alt}_{k}$.

Assume that $k \geq 8$. By Nag52, we have that there exists a prime number $p$ such that $k / 2<p<k-2$. By Theorem [26, we have that $p$ divides $|Z|$, hence $Z$ contains a cycle of order $p$. Since $Z$ is a primitive group of degree $k$ containing a cycle of order $p$, by [Jor73, Théorème I] we have that $Z \geq$ Alt $_{k}$ or $k \leq p+2$. Since $p<k-2$ we get that $Z$ contains $\mathrm{Alt}_{k}$ and the proof is complete.

Theorem 29. Let $G$ and $H$ be two finite groups such that $P_{G}(s)=P_{H}(s)$. Assume that the composition factors of $G$ and $H$ are alternating groups which satisfy the hypothesis of Theorem 2 . Then $G$ and $H$ have the same non-Frattini chief factors.

Proof. By [Pat10, Theorem 1] we have that $G$ and $H$ have the same non-Frattini abelian chief factors. So, we may assume that $G$ and $H$ have no non-Frattini abelian chief factors.

Let $\mathcal{C F}(G)$ be the set of non-Frattini chief factors of $G$. For $A \in \mathcal{C F}(G)$, let $L_{A}$ be the primitive monolithic group associated with $A$, defined by $L_{A}=G / C_{G}(A)$ (note that $A \cong \operatorname{soc}\left(L_{A}\right)$, for reference see [DL03, Definition 8] Pat10, Section 2]).

By [DL03, Theorem 18] we have that

$$
P_{G}(s)=\prod_{A \in \mathcal{C} \mathcal{F}(G)} \tilde{P}_{L_{A}, A}(s),
$$

where $\tilde{P}_{L_{A}, A}(s)$ are suitable polynomial such that $\tilde{P}_{L_{A}, A}^{(r)}(s)=P_{L_{A}, A}^{(r)}(s)$ for each $r \in \mathrm{p}(A)$. So, $\tilde{P}_{L_{A}, A}(s)$ is irreducible since in Theorem 23 and Proposition 24 we proved that a Dirichlet polynomial $P(s)$ such that $\mathrm{p}(A)=\left\{r: \exists k\left(a_{k}(P(s)) \neq 0, r \mid\right.\right.$ $k)\}$ and $P^{(r)}(s)=P_{L_{A}, A}^{(r)}(s)$ for each $r \in \mathrm{p}(A)$ is irreducible.

Thus there exists a bijection between the sets $\mathcal{C F}(G)$ and $\mathcal{C F}(H)$ such that $A \in \mathcal{C F}(G)$ and $B \in \mathcal{C F}(H)$ are associated if and only if $\tilde{P}_{L_{A}, A}(s)=\tilde{P}_{L_{B}, B}(s)$. We claim that $A \cong B$. Since $P_{L_{A}, A}^{(r)}(s)=P_{L_{B}, B}^{(r)}(s)$ for each $r \in \mathrm{p}(A)$, by Proposition 27 we have that $n=\left|L_{A}: N_{L_{A}}\left(S_{A}\right)\right|=\left|L_{B}: N_{L_{B}}\left(S_{B}\right)\right|$, hence we get $P_{X_{A}, S_{A}}^{(r)}(s)=$ $P_{X_{B}, S_{B}}^{(r)}(s)$ for each $r \in \mathrm{p}\left(S_{A}\right)$. By Proposition 28 , this implies that $S_{A} \cong S_{B}$, hence $A \cong S_{A}^{n} \cong S_{B}^{n} \cong B$.

\section{REFERENCES}

[Bos96] N. Boston. A probabilistic generalization of the Riemann zeta function. Analytic Number Theory, 1:155-162, 1996. MR1399336 (97e:11106)

[Che10] Yuan-You Fu-Rui Cheng. Explicit estimate on primes between consecutive cubes. Rocky Mountain J. Math., 40(1):117-153, 2010. MR2607111(2011f:11120)

[DL03] E. Detomi and A. Lucchini. Crowns and factorization of the probabilistic zeta function of a finite group. J. Algebra, 265:651-668, 2003. MR.1987022 (2004e:20119)

[DL07] E. Damian and A. Lucchini. Finite groups with p-multiplicative probabilistic zeta function. Comm. Algebra, 35(11):3451-3472, 2007. MR2362665 (2008k:20045)

[DLM04] E. Damian, A. Lucchini, and F. Morini. Some properties of the probabilistic zeta function of finite simple groups. Pacific J. Math., 215:3-14, 2004. MR2060491 (2005b:20042)

[Dus10] P. Dusart. Estimates of some function over primes without R.H. 2010. 
[Erd34] P. Erdös. A theorem of Sylvester and Schur. J. London Math. Soc., S1-9 no. 4, 282-288, 1934 .

[GAP] The GAP Group. GAP - Groups, Algorithms, and Programming, Version 4.4.12.

[Hal36] P. Hall. The Eulerian functions of a group. Quart. J. Math., 7:134-151, 1936.

[HIÖ89] T. Hawkes, M. Isaacs, and M. Özaydin. On the Möbius function of a finite group. Rocky Mountain Journal, 19:1003-1034, 1989. MR.1039540 (90k:20046)

[Jor57] C. Jordan. Traité des substitutions et des équations algébriques - Nouveau tirage de l'édition de 1870. Gauthier-Villars, A. Blanchard, Paris, 1957. MR0091260 (19:937c)

[Jor73] C. Jordan. Sur la limite de transitivité des groupes non alternés. Bull. Soc. Math. France, 40-71, 1872-73. MR 1503635

[Lan02] S. Lang. Algebra, volume 211. Graduate Texts in Mathematics, New York, 2002. MR.1878556 (2003e:00003)

[Leh64] D. H. Lehmer. On a problem of Störmer. Ill. J. Math., 8:57-79, 1964. MR0158849 $(28: 2072)$

[LP67] L. J. Lander and T. R. Parkin. On the first appearance of prime differences. Math. Comp., 21:483-488, 1967. MR0230677(37:6237)

[LPS90] M. Liebeck, C. Praeger, and J. Saxl. The maximal factorization of the finite simple groups and their automorphism groups. Mem. Amer. Math. Soc. 86 (1990), no. 432, Providence, Rhode Island. MR 1016353 (90k:20048)

[Man96] A. Mann. Positively finitely generated groups. Forum Math., 8:429-459, 1996. MR1393323 (97j:20029)

[Mas07] M. Massa. The probabilistic zeta function of the alternating group $\operatorname{Alt}(p+1))$. Boll. Unione Mat. Ital. Sez. B Artic. Ric. Mat., 8:581-591, 2007. MR2351530(2009c:20038)

[Nag52] J. Nagura. On the interval containing at least one prime number. Proc. Japan Acad., 28:177-181, 1952. MR0050615 (14:355b)

[NN03] Bertil Nyman and Thomas R. Nicely. New prime gaps between $10^{15}$ and $5 \times 10^{16}$. J. Integer Seq., 6:Article 03.3.1, 2003. MR1997838 (2004e:11143)

[Pat09] M. Patassini. On the irreducibility of the Dirichlet polynomial of a simple group of Lie type. Accepted by Israel J. Math.

[Pat10] M. Patassini. Recognizing the non-Frattini abelian chief factors from the Probabilistic Zeta function of a finite group. In preparation, 2010.

[Sch76] L. Schoenfeld. Sharper bounds for the Chebyshev functions $\varphi(x)$ ii. Math. Comput., 30(134):337-360, 1976. MR0457374 (56:15581b)

[Ser08] P. Jiménez Seral. Coefficient of the Probabilistic Zeta function of a monolithic group. Glasgow J. Math, 50:75-81, 2008. MR2381734(2008j:20066)

[Sta97] R. P. Stanley. Enumerative Combinatorics, Vol. 1. Cambridge University Press, Cambridge, 1997. MR1442260 (98a:05001)

Dipartimento di Matematica, Università di Padova, Via Trieste, 63 - 35121 Padova, ITALIA

E-mail address: frapmass@gmail.com 